\title{
A Phosphate-Dependent Requirement for Transcription Factors IPD3 and IPD3L During Arbuscular Mycorrhizal Symbiosis in Medicago truncatula
}

\author{
Penelope L. Lindsay, ${ }^{1,2}$ Brandon N. Williams, ${ }^{1}$ Allyson MacLean, ${ }^{1}$ and Maria J. Harrison ${ }^{1, \dagger}$ \\ ${ }^{1}$ Boyce Thompson Institute, Tower Road, Ithaca, NY 14853 \\ ${ }^{2}$ School of Integrative Plant Science, Plant Biology Section, Cornell University, Ithaca, NY \\ Accepted 7 May 2019.
}

\begin{abstract}
During arbuscular mycorrhizal (AM) symbiosis, activation of a symbiosis signaling pathway induces gene expression necessary for accommodation of AM fungi. Here, we focus on pathway components Medicago truncatula INTERACTING PROTEIN OF DOES NOT MAKE INFECTIONS 3 (IPD3) and IPD3 LIKE (IPD3L), which are potential orthologs of Lotus japonicus $\mathrm{CY}$ CLOPS, a transcriptional regulator essential for AM symbiosis. In the double mutant ipd3 ipd3l, hyphal entry through the epidermis and overall colonization levels are reduced relative to the wild type but fully developed arbuscules are present in the cortex. In comparison with the wild type, colonization of ipd3 ipd $3 l$ is acutely sensitive to higher phosphate levels in the growth medium, with a disproportionate decrease in epidermal penetration, overall colonization, and symbiotic gene expression. When constitutively expressed in ipd 3 ipd $3 l$, an autoactive DOES NOT MAKE INFECTIONS 3 induces the expression of transcriptional regulators REDUCED ARBUSCULAR MYCORRHIZA 1 and REQUIRED for ARBUSCULE DEVELOPMENT 1, providing a possible avenue for arbuscule development in the absence of IPD3 and IPD3L. An increased sensitivity of ipd3 ipd3l to $\mathrm{GA}_{3}$ suggests an involvement of DELLA. The data reveal partial redundancy in the symbiosis signaling pathway, which may ensure robust signaling in lowphosphorus environments, while IPD3 and IPD3L maintain signaling in higher-phosphorus environments. The latter may buffer the pathway from short-term variation in phosphorus levels encountered by roots during growth in heterogeneous soil environments.
\end{abstract}

Keywords: mycorrhizal interactions

${ }^{\dagger}$ Corresponding author: M. J. Harrison; mjh78@ cornell.edu

Funding: This research was supported by the United States National Science Foundation (US NSF) IOS 1353367. Confocal images were taken in the BTI Plant Cell Imaging Center, which received support from US NSF Instrumentation Grant DBI 0618969. P. L. Lindsay was supported by the United States Department of Agriculture-National Institute of Food and Agriculture Predoctoral Fellowship 2017-67011-26032. B. N. Williams participated in this research through a US NSF Plant Genome REU program, supported by US NSF DBI 1358843.

*The $\boldsymbol{e}$-Xtra logo stands for "electronic extra" and indicates that 11 supplementary figures and four supplementary tables are published online.

The author(s) declare no conflict of interest.

@ 2019 The American Phytopathological Society
For many land plants, access to phosphorus (P), an essential mineral nutrient, is greatly enhanced through a mutualistic endosymbiosis with arbuscular mycorrhizal (AM) fungi (Smith and Read 2010). In these associations, the fungi transfer phosphate (Pi) to root cortical cells in exchange for carbon (MacLean et al. 2017). Development of the symbiosis is negatively correlated with the plant's $\mathrm{P}$ status and this manifests at several levels; the number of infection points, the ability of the fungus to grow through the epidermis (Mosse 1973), extension within the cortex, and the frequency of arbuscules all generally decrease as plant $\mathrm{P}$ levels increase, although there is variation in these responses in different AM symbioses (Amijee et al. 1989; Thomson et al. 1986). Prolonged growth in high-P conditions decreases the production and secretion of strigolactones, symbiotic signaling molecules that promote presymbiotic fungal growth and initial contact with the root (Akiyama et al. 2005; Balzergue et al. 2011; Buee et al. 2000; Tawaraya et al. 1998). This, at least in part, explains the effects of high-P status on the number of primary infections and possibly extension in the cortex (Kobae et al. 2018) but not on other aspects of the symbiosis (Balzergue et al. 2013; Breuillin et al. 2010).

Development of AM symbiosis includes two stages that require intracellular growth of the fungus; the first occurs at the root epidermis and the second in the cortex. At the epidermis, fungal hyphae swell to form hyphopodia and penetrating hyphae subsequently grow through the epidermal cells into the root. In the root cortex, the hyphae penetrate cortical cells, where they differentiate to form arbuscules, the sites of nutrient exchange with the plant (Gutjahr and Parniske 2013; Lanfranco et al. 2018).

Development of the symbiosis and, in particular, intracellular hyphal growth is controlled by a signaling pathway referred to as the common symbiosis signaling pathway (CSSP) because, in legumes, it is also required for root nodule symbiosis (RNS) (Oldroyd 2013). Signaling through the pathway is triggered by lipochitooligosaccharides (Maillet et al. 2011) and short-chain chitooligosaccharide molecules (Genre et al. 2013) that are perceived by LysM-domain receptor kinases, although the full complement of receptors functioning in AM symbiosis has yet to be identified (Miyata et al. 2014; Zhang et al. 2015a). In Medicago truncatula, downstream signaling requires DOES NOT MAKE INFECTIONS 2 (DMI2), a leucine-rich receptor-like kinase which may function as a coreceptor (Bradbury et al. 1991; Endre et al. 2002). DMI2 interacts with HMGR coreductase and it is proposed that mevalonate transmits the signal from the plasma membrane to the nucleus (Venkateshwaran et al. 2015). Here, 
activation of cyclic nucleotide-gated CNGC15 channels together with a potassium-permeable ion channel, DOES NOT MAKE INFECTIONS 1 (DMI), resulting in sustained oscillations of calcium levels in the nucleus, known as calcium spiking (Charpentier et al. 2016; Riely et al. 2007; Saito et al. 2007). In Lotus japonicus, the role of DMI1 is carried out by two ion channels, CASTOR and POLLUX (Charpentier et al. 2008; Kistner et al. 2005). In both legume species, the calcium signal is then further transduced by a calcium-dependent protein kinase $M$. truncatula DOES NOT MAKE INFECTIONS 3 (DMI3)/L. japonicus calcium- and calmodulin-dependent protein kinase (CCaMK). The MtDMI3/LjCCaMK protein consists of a kinase domain, a calmodulin-binding domain, and an autoinhibitory EF-hand domain that chelates calcium (Singh and Parniske 2012). Upon calcium binding to the EF-hand domain, a conformational change is induced, autophosphorylating MtDMI3/LjCCaMK, which allows it to phosphorylate other protein substrates. MtDMI3/LjCCaMK phosphorylates at least one protein, $M$. truncatula INTERACTING PROTEIN OF DMI3 (IPD3)/L. japonicus CYCLOPS, a coiled-coil domain transcription factor that is constitutively bound to MtDMI3/LjCCaMK (Messinese et al. 2007; Yano et al. 2008). In L. japonicus, phosphorylation of LjCYCLOPS by LjCCaMK allows it to form a homodimer and bind to symbiosis-specific promoter elements, inducing the expression of several genes necessary for either AM symbiosis or RNS, including REDUCED ARBUSCULAR MYCORRHIZA 1 (LjRAM1), LjNIN1, and LjERN1 (Cerri et al. 2017; Pimprikar et al. 2016; Singh et al. 2014).

Although not a member of the CSSP, DELLA, a transcriptional regulator and repressor of gibberellic acid (GA) signaling, also influences expression of genes required for $\mathrm{AM}$ symbiosis. There is evidence for action downstream of CCaMK and independent of CYCLOPS but also for action through an association with CCaMK and CYCLOPS (Floss et al. 2013a; Jin et al. 2016; Pimprikar et al. 2016).

Most CSSP mutants display defects in AM fungal entry into epidermal or cortical cells, indicating the importance of the CSSP for intracellular hyphal growth. In $d m i 1, d m i 2$, and dmi3, epidermal entry is blocked (Bradbury et al. 1991; Endre et al. 2002; Lévy et al. 2004; Riely et al. 2007). L. japonicus orthologs of these genes display mutant phenotypes very similar to those reported in $M$. truncatula (Kistner et al. 2005; Lévy et al. 2004; Pimprikar et al. 2016). In $M$. truncatula, cngc 15 mutants display a reduced colonization phenotype but functional redundancy has prevented analyses of a complete loss-of-function mutant (Charpentier et al. 2016).

In general, the core CSSP mutants display similar phenotypes regardless of the plant species but this is not true of cyclops/ipd3. In L. japonicus and rice, cyclops mutants show a strong AM symbiosis phenotype with a complete absence of arbuscule formation in rice and arbuscules present only very rarely in L. japonicus (Gutjahr et al. 2008; Yano et al. 2008). In contrast, the phenotype displayed by $M$. truncatula ipd $3 \mathrm{mu}-$ tants is considerably less severe. Horváth et al. (2011) described two ipd3 alleles: a fast-neutron allele, ipd3-1, and a Tnt1 insertion, ipd3-2. Although colonized ipd3-2 roots were reported to lack arbuscules, ipd3-1 displayed a quantitative reduction in colonization, with normal arbuscule development. This variability in mutant phenotypes was hypothesized to result from the genetic background, because ipd3-1 is an A17 mutant, whereas ipd3-2 in an R108 allele; however, the lack of arbuscules in ipd3-2 was not observed by other researchers (Floss et al. 2013a). A third M. truncatula ipd3 allele, Mtsym1-1/TE7 from the Jemalong background, displays a reduction in colonization but increased arbuscule abundance, akin to that reported for Pisum sativum sym33-3 (Jacobi et al. 2003; Ovchinnikova et al. 2011). SYM33-3 is an ortholog of IPD3. The severity of the phenotype does not seem to be related to the strength of the allele because the tomato $r m c$ mutant, which consists of a deletion of five genes, including CYCLOPS, also shows a quantitative reduction in colonization (Larkan et al. 2013).

A potential explanation for the substantial difference in phenotypes of the L. japonicus cyclops and M. truncatula ipd3 mutants was provided by the discovery of an IPD3 paralog in M. truncatula (Bravo et al. 2016). However, a recent report of the independent discovery of the IPD3 paralog, called IPD $3 L$, and analysis of an ipd 3 ipd $3 l$ double mutant indicated that the double mutant also supports wildtype (WT) arbuscule development (Jin et al. 2018). Building on the identification of the IPD3 paralog (Bravo et al. 2016), we also generated an ipd3 ipd3l double mutant to test the hypothesis that IPD3L contributes to AM symbiotic signaling. Consistent with Jin et al. (2018), we find that our ipd3 ipd $3 l$ double mutant is capable of symbiotic development indicating, in contrast to the situation in L. japonicus, that the two IPD3 transcription factors are not essential for AM symbiosis. The nonessential role for IPD3 and IPD3L suggests that downstream signaling for AM symbiosis can proceed by other avenues, albeit less efficiently. However, the AM symbiosis phenotype of ipd3 ipd3l shows a heightened sensitivity to $\mathrm{Pi}$, with a greater reduction in the frequency of successful epidermal penetration events and overall colonization relative to the WT indicating a role for these proteins in maintaining signaling for AM symbiosis in different environments.

\section{RESULTS}

\section{M. truncatula has a paralog of IPD3, IPD3L.}

M. truncatula has a paralog of IPD3, named IPD3L (Bravo et al. 2016; Jin et al. 2018). In a phylogeny, IPD3L clusters independently of other legume homologs of IPD3 (Supplementary Fig. S1). This divergence is reflected in the amino acid sequence; IPD3 and its L. japonicus ortholog CYCLOPS share $80 \%$ amino acid identity, whereas IPD3 and IPD3L share $65 \%$ amino acid identity (Supplementary Fig. S2). All critical features previously described for CYCLOPS, including the nuclear localization signal and the serine residues necessary for activation by CCaMK (Singh et al. 2014), are conserved between IPD3 and IPD3L.

The IPD3L locus ID is Medtr8te071320 in M. truncatula genome version 4.0, which indicates that this coding sequence (CDS) is predicted to be contained within a transposable element (Young et al. 2011). A synteny analysis indicates that the regions of the genome containing LjCYCLOPS and MtIPD3 show a high degree of synteny (Supplementary Fig. S3). In contrast, the two genomic regions containing IPD3 and IPD3L are largely nonsyntenic. Together, these data, in combination with the fact that $M$. truncatula is the only plant species with a publicly available genome in which we could find a duplication of this gene, suggest that IPD $3 L$ may have arisen from a postspeciation transposon insertion event.

The $M$. truncatula genome version 4.0 predicts that $I P D 3 L$ contains an additional 588-bp exon at the $3^{\prime}$ end of the gene that is not present in IPD3. However, Softberry FGENESH predicts two separate CDS: one encoding a 506-amino-acid protein closely matching the IPD3 sequence and the second encoding a 116-amino-acid protein with a Ribonuclease Fold $\mathrm{H}$ motif and a reverse-transcriptase Zn-binding domain (Supplementary Fig. S4). A previous report indicated that $I P D 3 L$ contained this additional exon and that a full-length 
CDS including this C-terminal extension could be amplified and cloned from cDNA (Jin et al. 2018). In contrast, we were unable to amplify this extra exon from either mock-inoculated root cDNA or Rhizophagus irregularis-colonized root cDNA in either A17 or R108 ecotypes using primers that spanned the IPD3L exon 10 and the predicted an additional $3^{\prime}$ exon (exon 11) (Supplementary Fig. S5). A full-length IPD3L cDNA of 1,515 nucleotides was readily amplified from mycorrhizal root cDNA. The genomic and cDNA sequences are presented in Supplementary Figure S6. Consequently, we conclude that the extra predicted exon is not part of the IPD $3 L \mathrm{CDS}$.

$I P D 3 L$ transcripts were monitored by quantitative PCR (qPCR) in noncolonized and colonized roots from the WT R108 ecotype of $M$. truncatula. IPD3L transcript levels were similar in both samples (Fig. 1A and B) and were approximately six times lower than those of IPD3. In A17, IPD3 and $I P D 3 L$ show constitutive expression in noncolonized and colonized roots and, in this accession, transcript levels of IPD3 and IPD $3 L$ were similar to one another.

\section{Symbiont entry into epidermal cells is impaired in ipd3-2 ipd3l-2.}

To test the hypothesis that IPD3L functions redundantly with IPD3, we obtained Tnt1 transposon insertion alleles for IPD3 (NF5939) and IPD3L (NF13859) (Tadege et al. 2008) and generated a double mutant. The ipd3 allele in line NF5939 was described previously as ipd3-2 (Horváth et al. 2011). Our ipd3l Tnt1 allele differs from that described by Jin et al. (2018) and, therefore, is termed ipd3l-2. The Tnt1 insertion sites for both alleles are indicated in Figure 1C.

To assess the ipd3-2 ipd3l-2 AM symbiosis phenotype, seedlings were planted into substrate containing Glomus versiforme spores, fertilized with $1 / 2 \times$ Hoagland's containing $20 \mu \mathrm{M} \mathrm{Pi}$, and harvested 3 weeks postplanting. Under these conditions, colonization levels in the single mutants did not differ significantly from those of the parental WT R108 or WT segregant; however, there was a $50 \%$ reduction in colonized root length in ipd3-2 ipd3l-2 relative to WT (Fig. 1D). In the mutants, intercellular hyphae and arbuscules were visible in the cortex and were morphologically indistinguishable from those in WT roots (Fig. 1F and G). This is consistent with the report of Jin et al. (2018), who evaluated the mutants with $R$. irregularis. In contrast, there was a visible difference between WT and ipd3-2 ipd3l-2 at the epidermis, where hyphopodia that had failed to penetrate the roots were more abundant in ipd3-2 ipd3l-2 (Fig. 1H); the frequency of successful hyphal entry through the epidermis was $50 \%$ lower in ipd3-2 ipd3l-2 relative to the WT (Fig. 1E). Based on these data, the reduction in colonized root length in ipd3-2 ipd $3 l-2$ is likely the result of a reduction in epidermal penetration. Transcript levels of $G$. versiforme $\alpha$-tubulin and three $M$. truncatula AM symbiosis marker genes (PT4, VPY, and $R A M 1)$ were also reduced in ipd3-2 ipd3l-2 relative to the WT (Supplementary Fig. S7), consistent with the reduction in overall root colonization (Fig. 1D). The single mutants did not differ from WT, except for ipd3-2, which showed a reduction in $G$. versiforme $\alpha$-tubulin and PT4 transcripts. In this experiment, transcript levels in the WT segregant were lower than the parental WT (R108), even though colonized root length did not differ (Fig. 1D).

A growth response experiment was conducted to assess the long-term effect of the absence of IPD3 and IPD3L on plant fitness. Although both WT and ipd3-2 ipd3l-2 plants exhibited an increase in shoot biomass in colonized versus noncolonized conditions, there was no increase in number of seed pods in ipd3-2 ipd3l-2 colonized with G. versiforme, indicating that, although the mutant can be colonized, the symbiosis is not fully functional (Fig. 1I and J).

Because IPD3 is a member of the common symbiosis signaling pathway, we wanted to determine whether ipd3-2 ipd $3 l-2$ was also impaired in nodulation. It was reported previously that ipd3-2 has a defect in rhizobial infection thread and symbiosome formation, although nodules still form and, in fact, form in increased numbers in certain M. truncatula mutant alleles, including ipd3-2 (Horváth et al. 2011; Ovchinnikova et al. 2011). This is in contrast to Ljcyclops, where infection thread formation is arrested, and few nodule primordia are formed (Yano et al. 2008). To evaluate the nodulation phenotype of the double mutant, plants were inoculated with Sinorhizobium meliloti, and the nodulation phenotype was assessed 23 and 28 days postinoculation (dpi). At harvest, ipd3-2 and ipd3-2 ipd3l-2 shoots were noticeably smaller than the other genotypes (Supplementary Fig. S8). Infection threads that traversed the full length of the root hair were present in the single mutants but were not observed in ipd3-2 ipd3l-2, and S. meliloti mCherry strain 1021 was not observed in the very small nodules present in ipd3-2 ipd3l-2 at 23 dpi. S. meliloti expressing nifHpr: green fluorescent protein $(G F P)$ was used to monitor bacteria in the nodules. At $28 \mathrm{dpi}$, nifHpr:GFP-containing nodules were not observed on ipd3-2 ipd3l-2 plants (Supplementary Fig. S9). Additionally, the increase in nodules observed previously in ipd3-2 (Horváth et al. 2011) was suppressed in ipd3-2 ipd3l-2, although the majority of nodules in both ipd3-2 and ipd3-2 ipd3l-2 were immature. These results vary slightly from those obtained by Jin et al. (2018), where they observed a complete lack of nodule formation in ipd3-2 ipd3l-1. These results indicate that both IPD3 and IPD3L are important for nodulation, and that the infection thread phenotype of ipd3-2 ipd3l-2 resembles that of cyclops, although the nodule development may progress slightly beyond the primordia stage as defined by the scheme outlined by Xiao et al. (2014).

Overall, our phenotypic analysis suggests that, although the nodulation phenotype displayed by L. japonicus cyclops and M. truncatula ipd3-2 ipd3l-2 is similar, the AM symbiosis mutant phenotypes diverge. In ipd3-2 ipd3l-2, epidermal penetration is impaired but arbuscule development is unaffected whereas arbuscules are almost entirely absent in Ljcyclops.

\section{Development of AM symbiosis in ipd3-2 ipd3l-2 shows a heightened sensitivity to $\mathrm{Pi}$.}

Because P levels systemically affect AM symbiosis, and the ipd3-2 ipd3l-2 phenotype was mild under low-Pi fertilizer application, we tested whether the mutant phenotype could be exacerbated in higher-Pi environments. To evaluate the AM symbiosis phenotype during growth in different Pi fertilization regimes, ipd3-2 ipd3l-2 was transplanted into inoculated substrate and fertilized twice a week with $1 / 2 \times$ Hoagland's containing $20 \mu \mathrm{M}, 200 \mu \mathrm{M}$, or $2 \mathrm{mM}$ Pi. Transcript levels of a Pi starvation marker, Mt4 (Burleigh and Harrison 1999), were much lower in the 2-mM Pi-treated roots compared with the 20$\mu \mathrm{M}$ Pi-treated roots, indicating the efficacy of this fertilizer regime (Fig. 2A).

At 3 weeks postplanting, colonization levels were lower in ipd3-2 ipd3l-2 compared with the WT but were not significantly affected by 20 versus $200 \mu \mathrm{M}$ Pi (Fig. 3A). However, when fertilized with $2 \mathrm{mM} \mathrm{Pi}$, ipd3-2 ipd3l-2 displayed an eightfold reduction in colonized root length as compared with the 20- $\mu \mathrm{M}$ Pi fertilizer regime (Fig. 3A). In contrast, the WT segregant showed only a twofold decrease in colonized root length in the $2-\mathrm{mM}$ versus $20-\mu \mathrm{M}$ Pi treatment. Hyphal entry through the epidermis was reduced fivefold from the highest 


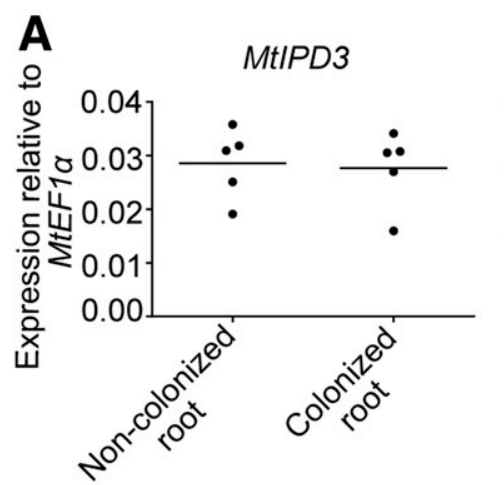

B

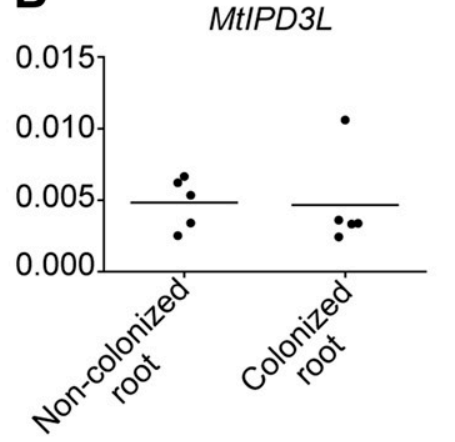

C

ipd3-2
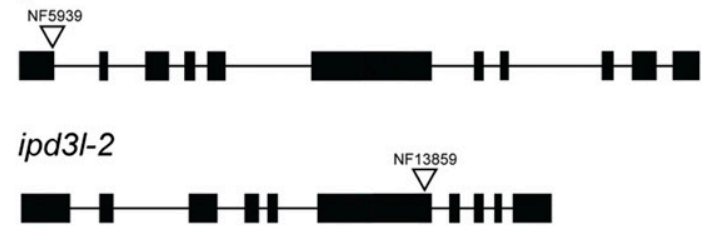

D

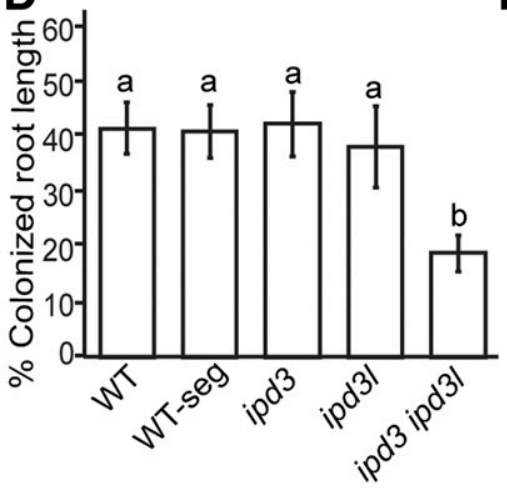

F

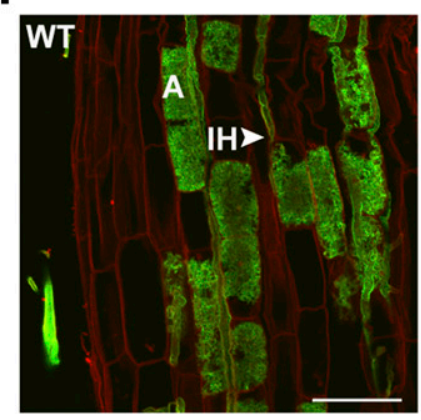

G

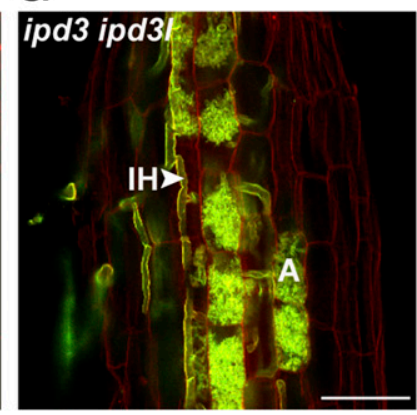

H

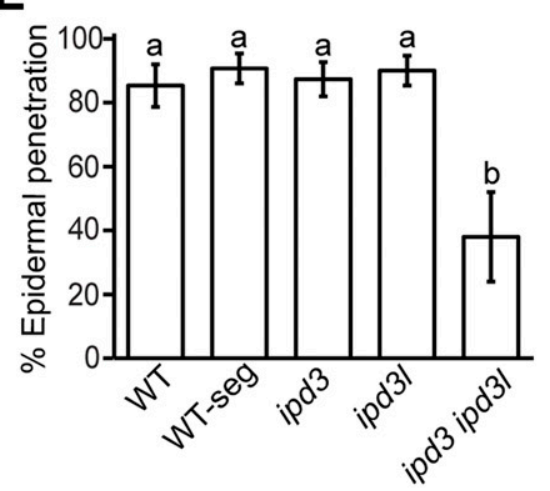

ipd3 ipd3I

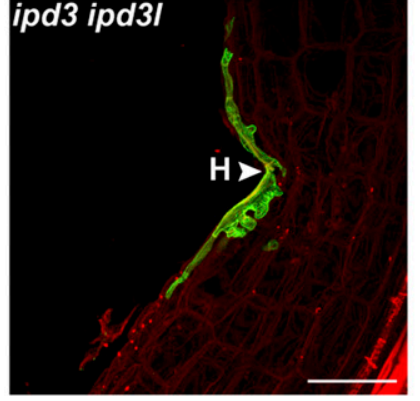

J

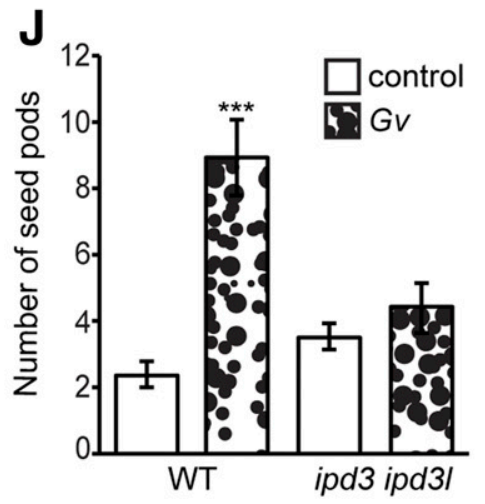

Fig. 1. IPD3 and IPD3L are necessary for efficient arbuscular mycorrhizal (AM) fungal infection. Gene expression of A, IPD3 and B, IPD3L in R108 Medicago truncatula noncolonized roots and roots colonized with Glomus versiforme; $n=5$. Bars indicate the mean. C, Gene structure of IPD3 (Medtr5g026850) and IPD3L (Medtr8g071320) showing the position of the Tnt1 insertions NF5939 (ipd3-2) and NF13859 (ipd3l-2) verified by sequencing. D, Percentage of colonized root length 3 weeks postplanting into $G$. versiforme inoculum; $n=7$. WT $=$ wild type, and letters indicate significant differences using analysis of variance (ANOVA) with $P<0.05$. E, Percentage of epidermal penetration by $G$. versiforme. Successful entries as a percentage of total hyphopodia are shown; $n=3$. One-way ANOVA with letters indicating statistically significant differences with $P<0.05$. Confocal images of $\mathbf{F}$, WT and $\mathbf{G}$ and $\mathbf{H}$, ipd3-2 ipd3l-2 roots colonized by $G$. versiforme. Plant cell wall material is stained with propidium iodide (red) and fungal cell walls are stained with WGA AlexaFluor488 (green). Scale bars are $50 \mu \mathrm{m}$. $\mathrm{H}=$ hyphopodium, IH = intercellular hyphae, A = arbuscule. I and $\mathbf{J}$, Growth response experiment. Plants were grown in soil substrate with $G$. versiforme or without $G$. versiforme (control) for 10 weeks to assess whether $G$. versiforme improves the growth and seed set of ipd3-2 ipd3l-2; $n=8$. Student's $t$ test comparing control versus $G$. versiforme, asterisks $* * *$ and $* *$ indicate a significance of $P<0.001$ and $<0.01$, respectively. 
to lowest Pi treatment in ipd3-2 ipd3l-2, whereas the WT segregant showed no change in epidermal entry over the three different Pi regimes (Fig. 3B; Supplementary Fig. S10). Additionally, in the 2-mM Pi condition, ipd3-2 ipd3l-2 displayed a $50 \%$ reduction in the number of infection units containing arbuscules, although the percentage of infection units containing arbuscules varied considerably between root systems because the total number of infection units was low (Fig. 3D). In the 2-mM Pi treatment, there was no statistically significant reduction in colonized root length with either of the single mutants but a $30 \%$ decrease in epidermal entry in ipd3-2 as compared with the WT segregant. The number of infection units and percentage of infection units containing arbuscules was not affected in either of the single mutants.

In addition, we measured the transcript levels of AM symbiosis marker genes. Consistent with the colonization data, transcript levels of AM symbiosis marker genes were much lower in ipd3-2 ipd3l-2 at $2 \mathrm{mM}$ Pi relative to $20 \mu \mathrm{M} \mathrm{Pi}$ (Fig. 2B to F). PT4 and RAM1 transcripts were almost undetectable in ipd3-2 ipd3l-2 plants under the 2-mM Pi fertilizer regime but there was no additional reduction in expression of $V P Y$ in ipd3-2 ipd $3 l-2$ relative to the WT in the high-Pi treatment. NSP1 transcript levels were affected only by the Pi treatment and did not differ between genotypes (Fig. 2F).

Thus, the colonization defects in ipd3-2 ipd3l-2 are more prominent at high Pi. Notably, epidermal entry decreases over fivefold in ipd3-2 ipd3l-2 at $2 \mathrm{mM}$ Pi relative to $20 \mu \mathrm{M} \mathrm{Pi}$, whereas the WT does not show a significant difference in epidermal entry between these two conditions (Fig. 3). The colonization data are further corroborated with the AM symbiosis gene transcripts of the WT segregant and ipd3-2 ipd3l-2 under $20-\mu \mathrm{M}$ and 2-mM Pi fertilizer regimes (Fig. 2). Because the Pi starvation marker $M t 4$ shows similar transcript levels in the WT and ipd3-2 ipd3l-2 at $20 \mu \mathrm{M}$ and $2 \mathrm{mM} \mathrm{Pi}$ (Fig. 2A), we conclude that the double mutant is not showing a general increase in sensitivity to $\mathrm{P}$ but, rather, a symbiosis-specific response.

\section{Overexpression of autoactive $I P D 3$ and $I P D 3 L$ induces downstream AM symbiosis gene expression in the absence of $\mathrm{AM}$ fungi.}

In L. japonicus, CYCLOPS is essential for the expression of $R A M 1$, a major regulator of arbuscule development and, in cyclops mutants, arbuscules do not develop (Park et al. 2015; Pimprikar et al. 2016; Yano et al. 2008). In contrast, in M. truncatula ipd3-2 ipd3l-2, RAM1 is expressed, albeit at lower levels, and arbuscules fully develop. Consequently, we questioned whether IPD3 and IPD3L regulate expression of RAM1 and other regulators of AM symbiosis in M. truncatula. To evaluate this, we generated autoactive versions of IPD3 and IPD3L by substituting the two serines equivalent to those necessary for the activation of CYCLOPS by CCaMK with two aspartate residues (Singh et al. 2014). Transcript levels of several AM symbiosis regulators were then monitored in $M$. truncatula roots constitutively expressing the autoactive versions, IPD3-DD and IPD $3 L-D D$. In comparison with a $\beta$-glucuronidase control, constitutive expression of both IPD3$D D$ and $I P D 3 L-D D$ resulted in a significant increase in $R A M 1$, REQUIRED for ARBUSCULE DEVELOPMENT 1 (RADI), $N S P 1$, and $V P Y$ transcripts (Fig. $4 \mathrm{C}$ to F) but not NSP2 or DELLAI (Fig. 4G and $\mathrm{H}$ ). The level of $V P Y$ transcripts induced by IPD3-DD or IPD3L-DD is similar to that observed in a wellcolonized WT root, while the level of RAMI transcripts is an order of magnitude lower than that observed in well-colonized WT roots (Figs. 4 and 2D). This suggests that, although both IPD3 and IPD3L can induce the expression of several regulatory genes necessary for AM symbiosis, additional proteins may be necessary for robust induction of RAMI.

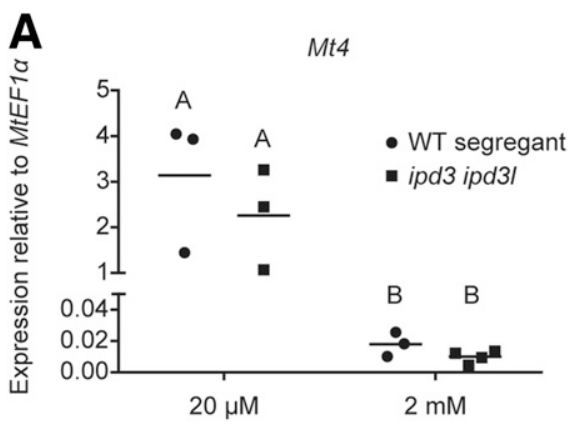

Phosphate concentration

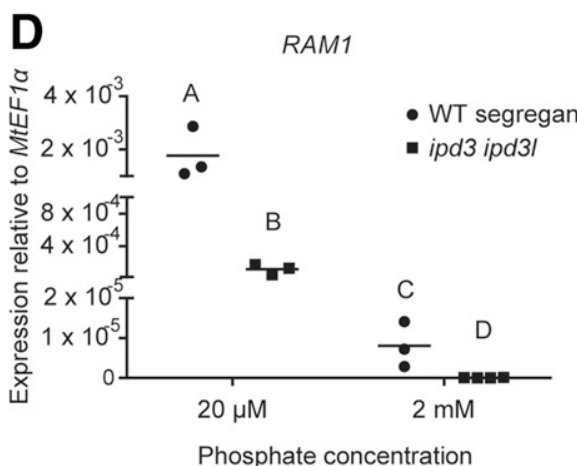

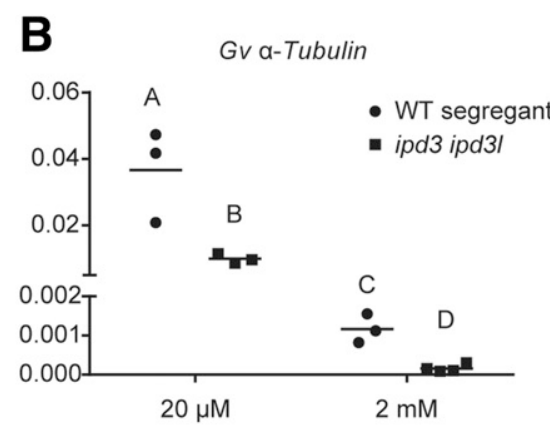

Phosphate concentration

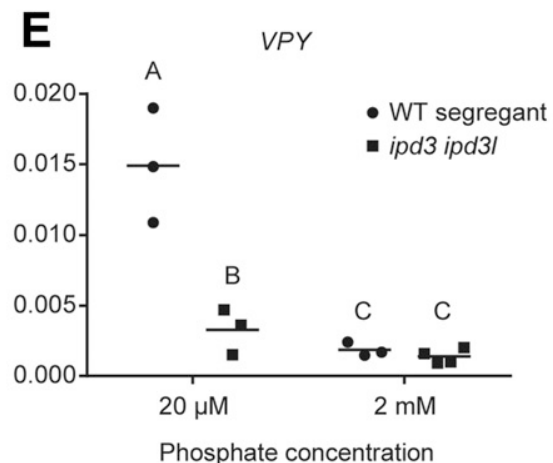

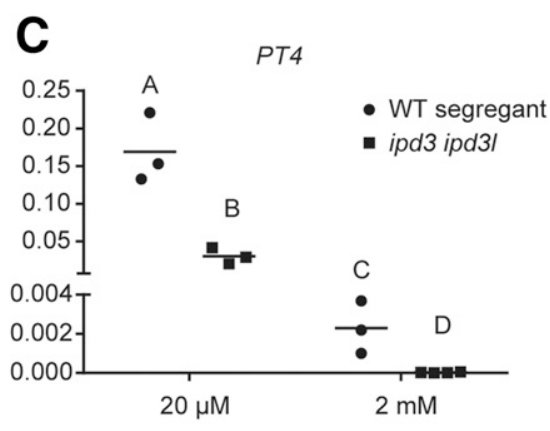

Phosphate concentration

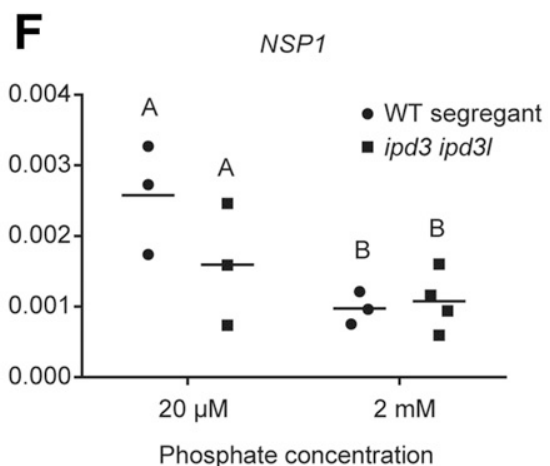

Fig. 2. Transcript levels of arbuscular mycorrhizal (AM) symbiosis genes in ipd3-2 ipd3l-2 under low and high phosphate conditions. Quantitative PCR was used to assess expression relative to Medicago truncatula elongation factor $1 \alpha$. A, Phosphate-starvation gene Mt4; $\mathbf{B}$, Glomus versiforme $\alpha$-tubulin; $\mathbf{C}$, PT4; D, RAM1; E, VPY; F, NSP1. Horizontal bars indicate the mean and points indicate individual replicates; $n \geq 3$. Different letters indicate statistically significant differences, $P<0.05$, using multiple comparisons $t$ test. 
Expression of $35 \mathrm{~S}$ :gain-of-function $\mathrm{DMI} 3$ induces AM symbiosis genes in an IPD3/IPD3L-dependent and IPD3/IPD3L-independent manner.

In L. japonicus, CYCLOPS is activated by CCaMK. CCaMK and its M. truncatula ortholog, DMI3, are essential for symbiosis and both L. japonicus ccamk and M. truncatula dmi3 mutants show a similar phenotype, in which the fungus fails to enter the root (Catoira et al. 2000; Lévy et al. 2004; Mitra et al. 2004). In L. japonicus, an autoactive version of CCaMK restores AM fungal infection events in ccamk (Takeda et al. 2012). Additionally, in the absence of a symbiotic signal, autoactive versions of CCaMK lacking its EF-hands but containing a nuclear localization sequence (NLS) stimulate AM symbiotic gene expression, although this version of the protein cannot fully complement ccamk (Takeda et al. 2012, 2015).

Building on the previous data, we asked whether an autoactive version of DMI3 requires IPD3 and IPD3L to induce expression of AM symbiosis genes. As a first step, we confirmed that IPD3L can interact with DMI3 in a yeast-twohybrid (Y2H) assay (Fig. 5A). Then, guided by the earlier analyses, we generated a gain-of-function (GOF) version of DMI3 lacking its autoinhibitory EF-hands with a phosphomimetic amino acid replacement of threonine 271 to aspartate (T271D) and a C-terminal NLS. In the absence of a symbiont, constitutive expression of GOF-DMI3-NLS in WT roots resulted in an increase in VPY, RAM1, RAD1, and NSP1 transcripts relative to roots expressing a $G F P$ control (Fig. 5B to E).
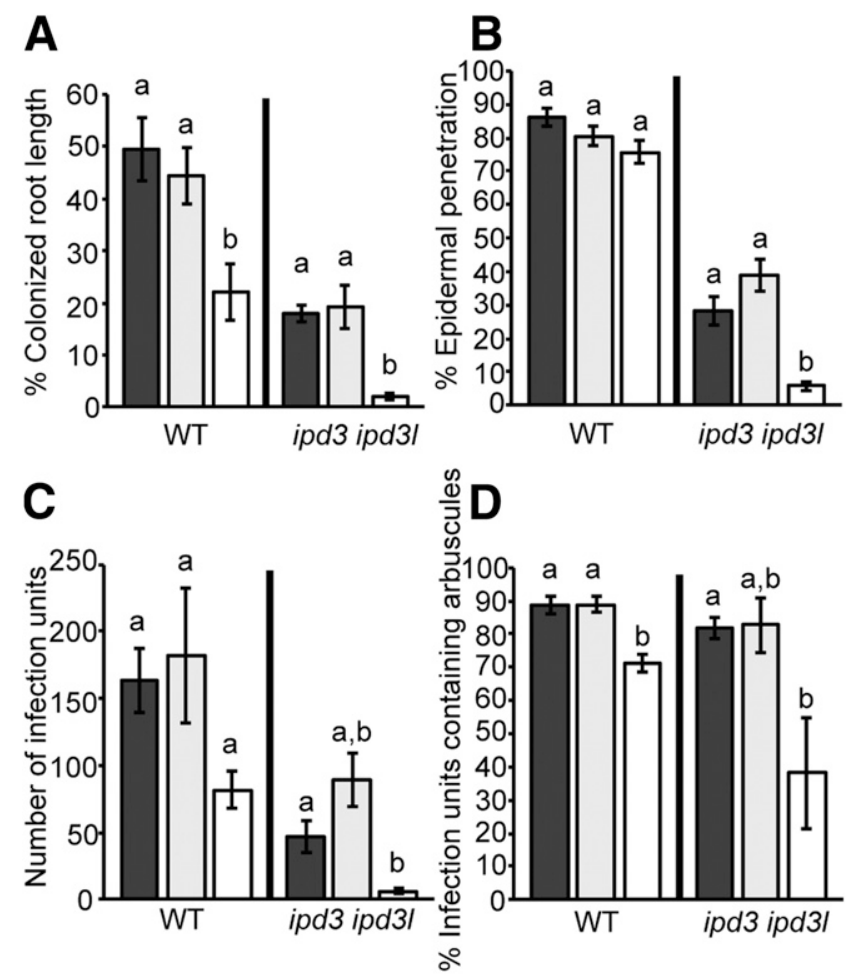

$20 \mu \mathrm{M} \square 200 \mu \mathrm{M} \square 2 \mathrm{mM}$

Phosphate concentration

Fig. 3. The ipd3-2 ipd3l-2 arbuscular mycorrhizal (AM) symbiosis phenotype is sensitive to high phosphate (Pi). Wild-type (WT) segregant and ipd3-2 ipd3l-2 plants were fertilized with $1 / 2 \times$ Hoagland's containing $20 \mu \mathrm{M}, 200 \mu \mathrm{M}$, or $2 \mathrm{mM} \mathrm{Pi}$ and $\mathbf{A}$, colonized root length; B, epidermal penetration; $\mathbf{C}$, number of infection units; and $\mathbf{D}$, percentage of infection units containing arbuscules were counted; $n=5$. Different letters indicate statistically significant differences using one-way analysis of variance within genotypes, Tukey's honestly significant difference.
In contrast, in ipd3-2 ipd3l-2 roots expressing GOF-DMI3$N L S, V P Y$ and NSP1 transcripts did not increase relative to the GFP controls. There was an increase in RAMI and $R A D I$ transcripts relative to the GFP controls, however (Fig. 5C and D), which was substantially lower than levels obtained in the WT.

The results of these two GOF experiments indicate that DMI3 signaling requires IPD3 and IPD3L for the transcriptional regulation of $V P Y, R A M 1, R A D 1$, and $N S P 1$ but they also indicate that signaling through DMI3 can trigger small increases in two major downstream regulators, RAM1 and $R A D 1$, in the absence of IPD3 or IPD3L.

\section{Arbuscule numbers increase in Ljcyclops-3 under low-Pi conditions.}

Because we could alter the ipd3-2 ipd3l-2 AM symbiosis phenotype by changing the $\mathrm{Pi}$ content of the growth environment, we wanted to determine whether the Ljcyclops phenotype could be affected by altering the growth conditions. Specifically, we were interested in whether arbuscule formation could be promoted if Ljcyclops was grown in low-Pi conditions, because it has previously been reported that arbuscules are rarely formed in Ljcyclops (Yano et al. 2008).

To test this, we grew WT and Ljcyclops-3 plants in G. versiforme-inoculated substrate and treated these plants with a fertilizer regime of $1 / 2 \times$ Hoagland's fertilizer containing either 0,20 , or $200 \mu \mathrm{M} \mathrm{Pi}$. We used a lower concentration gradient for $L$. japonicus than for $M$. truncatula because previous experiments in our standard $20-\mu \mathrm{M} \mathrm{Pi}$ growth conditions (Floss et al. 2013a) had shown that the Ljcyclops AM symbiosis phenotype is most similar to the "high-Pi" ipd3 ipd3l phenotype (Fig. 3). Colonization levels and arbuscule content were assessed in the WT and Ljcyclops at 5 weeks postplanting.

In all $\mathrm{Pi}$ growth conditions, the most prominent aspect of the Ljcyclops AM symbiosis phenotype was a failure to penetrate the epidermis. Many swollen hyphopodia were visible at the epidermis and intraradical colonization was reduced substantially (Fig. 6A to E). However, in all three Pi growth conditions, we observed roots with cortical infections that contained arbuscules; the highest number of arbuscules occurred in the $0-\mu \mathrm{M}-\mathrm{Pi}$ growth condition (Fig. $6 \mathrm{C}$ and $\mathrm{E}$ ). Thus, although cortical colonization and arbuscule development is severely impaired in Ljcyclops, a small phenotypic change (namely, an increase in arbuscule number) can be induced by decreasing Pi levels in the environment (Fig. $6 \mathrm{D}$ and E). However, this effect is small compared with the Pi-dependent phenotypic alterations observed in ipd 3 ipd $3 l$, and these experiments further support the hypothesis that there are differences in operation of AM symbiosis signaling in these two legumes.

\section{Hyphal entry through the epidermis is abolished in $\mathrm{GA}_{3}$-treated ipd3-2 ipd3l-2 plants.}

The Pi-dependent phenotype of ipd3-2 ipd3l-2, along with the results of the GOF-DMI3 experiments, led us to hypothesize that an additional factor enables symbiotic gene expression during growth in low-Pi conditions and, thus, partially compensates for the lack of IPD3 and IPD3L. DELLA proteins are possible candidates because they are required for AM symbiosis in M. truncatula (Floss et al. 2013a; Takeda et al. 2015) and can induce $R A M 1$ expression in the absence of CCaMK and CYCLOPS, although models from heterologous expression studies propose that a DELLA/CYCLOPS complex is needed for robust induction of symbiotic gene expression (Park et al. 2015; Pimprikar et al. 2016). 
It is well established that the phytohormone GA negatively regulates DELLA proteins by targeting them for degradation (Davière and Achard 2013; Floss et al. 2013a; Silverstone et al. 2001; Takeda et al. 2015) and application of GA allows manipulation of DELLA protein levels. Therefore, to test the hypothesis that DELLAs can compensate for a lack of IPD3 and IPD3L, WT segregant and ipd3-2 ipd3l-2 plants were inoculated with $G$. versiforme, fertilized with $1 / 2 \times$ Hoagland's containing $20 \mu \mathrm{M} \mathrm{Pi}$, and treated with $10^{-8}, 10^{-7}$, or $10^{-6} \mathrm{M}$ $\mathrm{GA}_{3}$ or a solvent solution lacking $\mathrm{GA}_{3}$.

Although there was no statistically significant change in infection unit number and epidermal penetration in the WT segregant with $10^{-6} \mathrm{M} \mathrm{GA}_{3}$, ipd3-2 ipd3l-2 epidermal penetration and infection unit formation was almost completely blocked (Fig. 7A and B). Many swollen hyphopodia were present on the root surface, indicating multiple entry attempts along the root and reminiscent of a dmi3 phenotype. At $10^{-8} \mathrm{M}$ $\mathrm{GA}_{3}$, the WT segregant displayed a $50 \%$ reduction in infection units containing arbuscules as compared with the solvent control but there was a $98 \%$ reduction in ipd3-2 ipd3l-2. This indicates that, in comparison with the WT, development of symbiosis is more sensitive to the application of GA in ipd3-2 ipd3l-2 (Fig. 7C).

It was previously demonstrated that constitutive expression of a dominant, GA-insensitive version of DELLA1, $D$ DELLA1, can partially complement the arbuscule defect in cyclops and increase colonization levels in ipd3 (Floss et al. 2013a). We confirmed that $35 \mathrm{~S}: \triangle D E L L A 1$ doubled the colonized root length of ipd3-2 ipd3l-2 as compared with expression of the negative control 35S:GFP, although the colonization did not reach the levels of the WT segregant.

If we assume that the GA treatment is influencing the presence of DELLA, these data suggest that DELLA, IPD3, and IPD3L influence epidermal entry and arbuscule formation in M. truncatula. Although IPD3 and IPD3L play a major role in AM fungal epidermal entry, we infer that DELLA1 may be able to induce a basal level of symbiotic gene expression to enable epidermal entry of AM fungi, because applying $\mathrm{GA}_{3}\left(10^{-6} \mathrm{M}\right)$ to ipd3-2 ipd3l-2 eliminates AM fungal epidermal entry. Conversely, DELLA may be playing a dominant but not exclusive role in arbuscule formation relative to IPD3 and IPD3L, because arbuscule formation is inhibited by a lower concentration of $\mathrm{GA}_{3}$ in ipd3-2 ipd3l-2 $\left(10^{-7} \mathrm{M}\right)$ as compared with the WT $\left(10^{-6} \mathrm{M}\right)$.

\section{DISCUSSION}

Building on the observation of an additional copy of IPD3 in the M. truncatula genome (Bravo et al. 2016), we generated a double mutant, ipd3-2 ipd3l-2, to evaluate the roles of these two genes in AM symbiosis. Although arbuscules were present, hyphal penetration of the epidermis and overall colonization levels were reduced in ipd3-2 ipd3l-2 relative to the WT, and this phenotype became disproportionately more severe in ipd3-2 ipd3l-2 when the Pi content of the growth medium was increased. From these data, two conclusions can
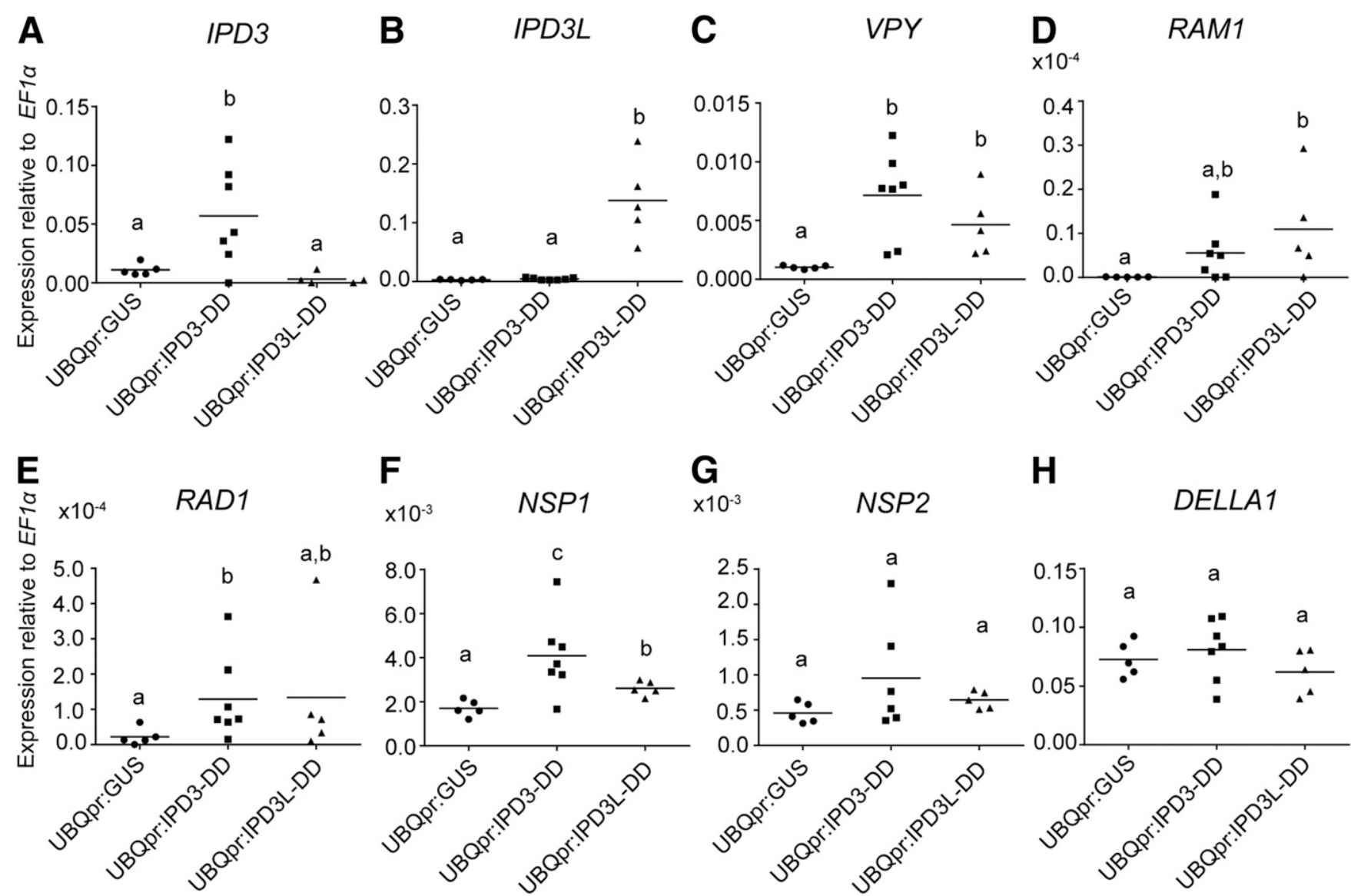

Fig. 4. Constitutive expression of autoactive versions of IPD3 and IPD3L results in an increase in transcript levels of several arbuscular mycorrhizal (AM) symbiosis genes in the absence of a fungal symbiont. A, IPD3; B, IPD3L; C, VPY; D, RAM1; E, RAD1; F, NSP1; G, NSP2; and H, DELLA1 transcripts were assessed in noncolonized transgenic roots containing either a control vector $M t U B Q$ promoter: $\beta$-glucuronidase (GUS) (UBQpr:GUS) or autoactive versions of IPD3 (UBQpr:IPD3-DD) and IPD3L (UBQpr:IPD3L-DD). Transcript levels relative to Medicago truncatula elongation factor 1 $\alpha(E F 1 \alpha)$; $n=5$. KruskalWallis test was performed to assess pairwise comparisons to UBQpr:GUS, different letters signify $P<0.05$, bars represent the mean. 
be drawn: first, there is redundancy or an alternate route through the CSSP when plants are highly $\mathrm{P}$ deprived; and second, IPD3 and IPD3L are essential for symbiosis signaling in higher-Pi environments. The physiological significance of the role of IPD3 and IPD3L during symbiotic signaling is supported by the growth response experiments in which inoculated ipd3-2 ipd3l-2 grown in soil showed no increase in fecundity as compared with uninoculated plants. An environmental effect on the phenotype of ipd3/cyclops mutants may also explain the wide variation reported in different plant species and mutant alleles, and also with differing fungal symbionts (Chen et al. 2008; Gutjahr et al. 2008; Horváth et al. 2011; Larkan et al. 2013; Ovchinnikova et al. 2011; Yano et al. 2008). However, the magnitude of Pi-dependent phenotypic change in Ljcyclops-3 is very small relative to that observed in ipd3 ipd3l; thus, it will be interesting to see whether the tomato cyclops displays a Pi-dependent shift in phenotype.

We demonstrated that fungal entry into the epidermis is reduced in ipd3-2 ipd3l-2, which is consistent with the accepted model that IPD3 interacts with DMI3 to initiate symbiotic gene induction (Hayashi et al. 2014; Pimprikar et al. 2016; Singh et al. 2014) and also with the dmi3 phenotype (Bradbury et al. 1991; Lévy et al. 2004). Furthermore, constitutive expression of autoactive variants of IPD3 and $I P D 3 L$ results in the expected transcriptional induction of several AM symbiosis genes, including VPY, a gene that influences epidermal penetration (Pumplin et al. 2010), thus consistent with the ipd3-2 ipd3l-2 epidermal phenotype.
Additionally, AM symbiosis gene expression induced by GOF-DMI3 largely requires IPD3 and IPD3L. However, the fact that the fungus can colonize ipd3-2 ipd3l-2 in low-Pi conditions indicates that there is another way to induce symbiotic gene expression in the absence of IPD3 and IPD3L that may be sensitive to high $\mathrm{Pi}$. The experiment in which an autoactive version of DMI3 was expressed constitutively in ipd3-2 ipd3l-2 provided initial clues suggesting that the answer may lie with the GRAS transcription factors RAM1 and $R A D 1$. Transcript levels of both genes increased in response to expression of GOF-DMI3 in ipd3-2 ipd3l-2, suggesting that $R A M 1$ and $R A D 1$ expression can occur independently of IPD3 and IPD3L, albeit at low levels. We hypothesized that this increase in transcripts might require DELLA proteins, whose expression is elevated in low-P conditions and whose protein levels are posttranslationally reduced in high-P conditions (Jiang et al. 2007; Floss et al. 2013a; Zentella et al. 2016). In support of this proposal, ectopic expression of a dominant DELLA in cyclops can induce the expression of RAMI and RAD1 and complements the arbuscule defect (Floss et al. 2013a; Park et al. 2015). Although DELLA has been proposed to operate in a complex with CYCLOPS and CCaMK, treatment of ccamk and cyclops with PAC, a GA inhibitor, results in increased expression of RAM1 (Park et al. 2015; Pimprikar et al. 2016), indicating an effect independent of the full complex. Furthermore, as shown here, colonization of ipd3-2 ipd3l-2 is more sensitive to $\mathrm{GA}_{3}$, with complete loss of cortical colonization at $10^{-6} \mathrm{MGA}_{3}$. This phenotype is consistent with the model that RAMI and RADI expression can be
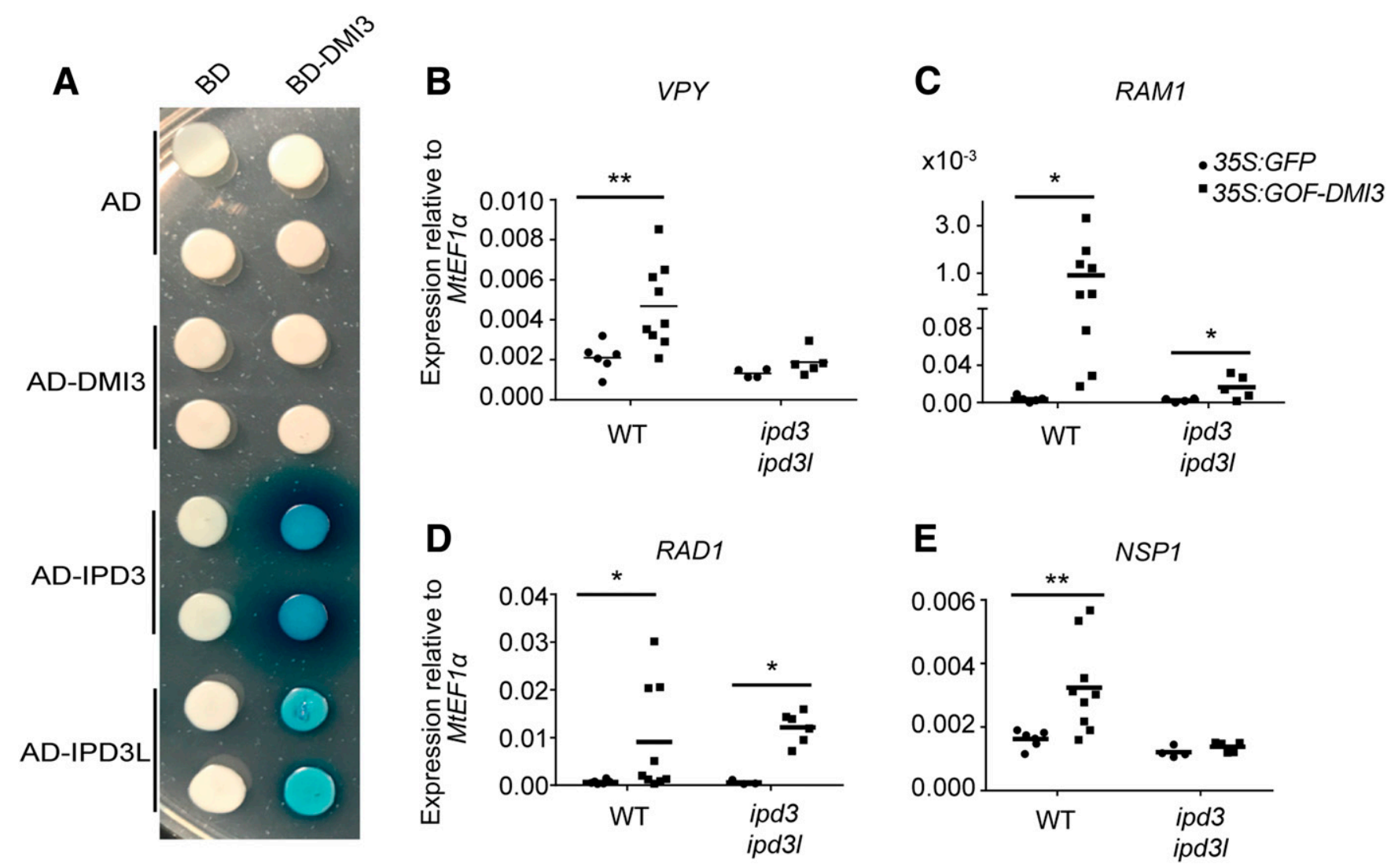

Fig. 5. IPD3 and IPD3L are necessary for the induction of symbiotic genes by autoactive DMI3 in the absence of a symbiont. A, IPD3L interacts with DMI3. In a yeast-two-hybrid assay, two separate mated yeast colonies were plated per interaction. As a positive control, the interaction between IPD3 and DMI3 was assayed. $\mathrm{AD}$ and $\mathrm{BD}=$ empty activation domain and binding domain vectors, respectively. Image taken after a 2 -day incubation at $28^{\circ} \mathrm{C}$. $\mathbf{B}$ to $\mathbf{E}$, An autoactive version of DMI3 was expressed under the CaMV35S promoter in wild-type (WT) and ipd3-2 ipd3l-2 noncolonized roots. Gene expression was relative to elongation factor $1 \alpha(E F 1 \alpha)$. Bars represent the mean, $n \geq 5$. Student's $t$ test was used to assess differences between control and gain-of-function (GOF)-DMI3. Asterisks $*$ and $* *$ indicate $P<0.05$ and 0.01 , respectively. 
induced by DMI3 and DELLA, enabling arbuscule development even in the absence of IPD3 and IPD3L. Thus, the CYCLOPS/CCaMK/DELLA complex proposed by Pimprikar et al. (2016) may incorporate redundancy that enables some signaling from just two of the three proteins. If this is correct, we would predict that other, as-yet-unknown genes that regulate epidermal entry will also be regulated in this manner, because epidermal penetration of ipd3-2 ipd3l-2 is likewise sensitive to $\mathrm{GA}_{3}$ (Fig. 7) and ectopic expression of a dominant DELLA increases the frequency of cortical infections of $c y$ clops (Floss et al. 2013a). Although the model is plausible, in our experiments, pleiotropic effects from exogenous $\mathrm{GA}_{3}$ application cannot be excluded at this time. We did attempt to complement the $2 \mathrm{mM}$ Pi ipd3-2 ipd3l-2 phenotype through expression of dominant DELLA; however, colonization was not observed, indicating that additional factors may be required (data not shown). To date, no attempts to complement the effect of high-Pi fertilizer on AM fungal colonization have been successful, perhaps because this systemic signal affects multiple factors necessary for AM symbiosis simultaneously (Balzergue et al. 2011, 2013; Branscheid et al. 2010).
In contrast to the differing AM symbiosis phenotypes of ipd3-2 ipd3l-2 and cyclops, their nodulation phenotypes are very similar, with premature arrest of infection thread formation and the inability to form mature nodules (Jin et al. 2018; Yano et al. 2008). Additionally, there appears to be some degree of nonoverlapping function between IPD3 and $I P D 3 L$ because the single mutant phenotypes are not strictly additive. This is shown by the fact that ipd3-2 has an increase in the number of nodules but this number is reduced in ipd3-2 ipd3l-2. Future work should address whether or not IPD3 and IPD3L have some divergence in the induction of specific symbiotic gene targets.

Due to the nutrient dependence of AM symbiosis, regulatory inputs that influence gene expression quantitatively dependent on the environment might be expected. Beyond the CSSP, many genes necessary for AM symbiosis contain the P1BS Pi starvation response promoter element, indicating that transcription factors activated when plants are P starved may help increase AM fungal colonization levels (Delaux et al. 2014).

In conclusion, this study reveals quantitative regulation of symbiotic development at the level of CSSP signaling and
A

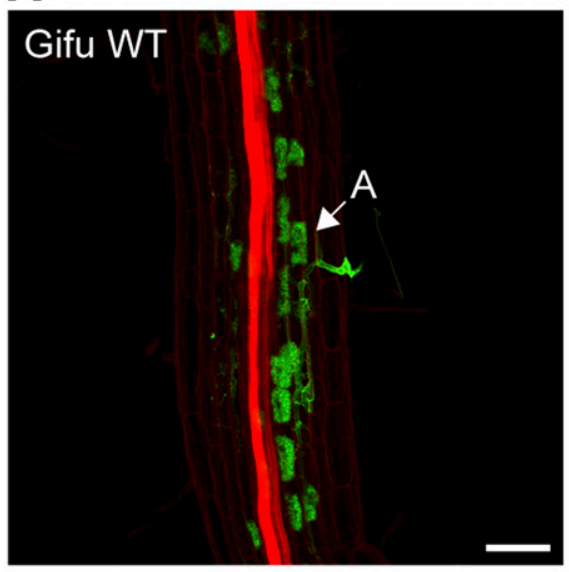

D

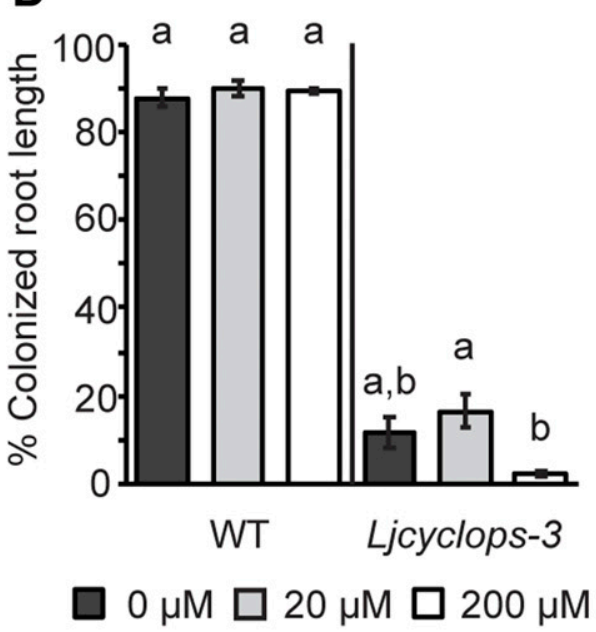

Phosphate concentration
B

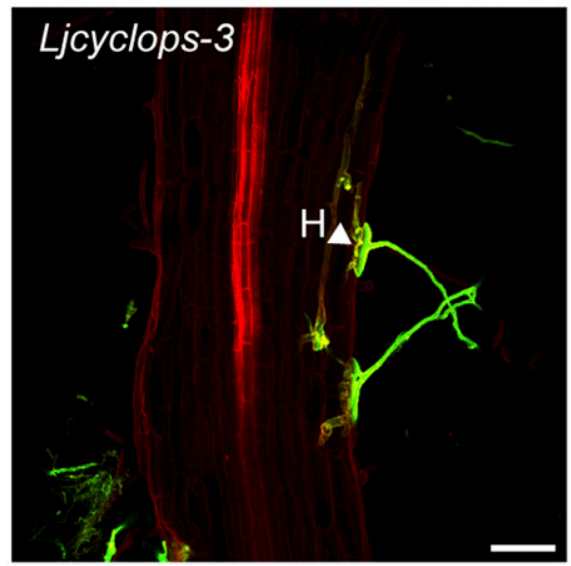

E

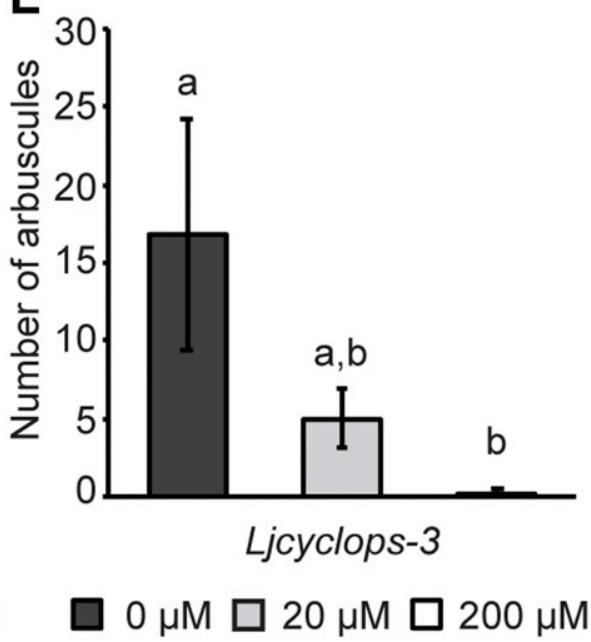

Phosphate concentration

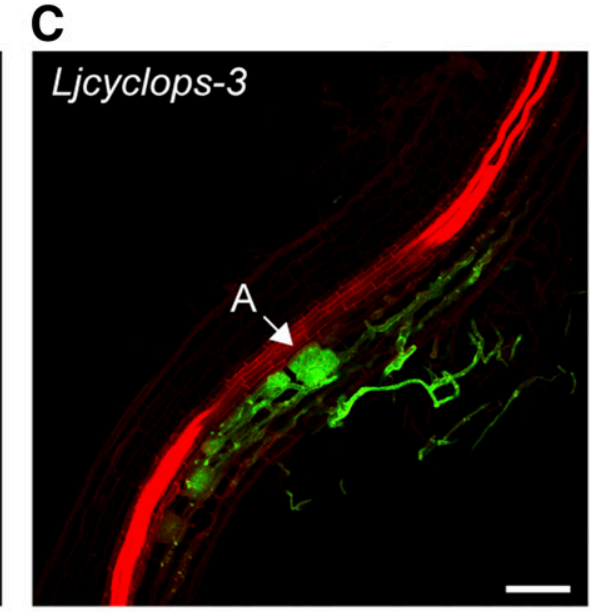

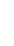


that the CSSP has some built-in redundancy that ensures signaling in low-P conditions. Additionally, we uncovered an essential role for IPD3 and IPD3L in maintaining CSSP signaling in higher-P environments. In a heterogeneous soil environment, this may be important and could potentially enable roots to maintain signaling as they encounter patches of soil with differing $\mathrm{P}$ levels.

\section{MATERIALS AND METHODS}

\section{M. truncatula plant growth and inoculation with AM fungi.}

For the ipd3-2 ipd3l-2 mutant phenotyping, M. truncatula seed were surface sterilized (Liu et al. 2004) and planted in 20.5-cm cones containing a 2:2:1 mixture of play sand (average particle size 200 to $300 \mu \mathrm{m}$ ), black sand (heterogeneous particle size $200 \mu \mathrm{m}$ to $5 \mathrm{~mm}$ ), and gravel (heterogeneous particle size $3 \mathrm{~mm}$ to $1 \mathrm{~cm}$ ), respectively, with 200 surface-sterilized $G$. versiforme spores (Liu et al. 2004) added at a depth of $3.5 \mathrm{~cm}$ into the cone, unless otherwise indicated. Two plants were planted per cone. Plants were transferred to a growth chamber with $16 \mathrm{~h}$ of light at $25^{\circ} \mathrm{C}$ and $8 \mathrm{~h}$ of darkness at $22^{\circ} \mathrm{C}$ and fertilized with $20 \mathrm{ml}$ of $1 / 2 \times$ Hoagland's, $1 / 2 \times$ nitrogen $(\mathrm{N})$, and $200 \mu \mathrm{M}$ potassium phosphate the day after planting, and subsequently fertilized with $1 / 2 \times$ Hoagland's, $1 / 2 \times \mathrm{N}$, and $20 \mu \mathrm{M}$ potassium phosphate twice a week, unless otherwise indicated. At 3 weeks postplanting, roots in the $6-\mathrm{cm}$ zone surrounding the spore layer were collected for gene expression analysis and staining.

For the growth response experiment, four seedlings were planted per pot in 11-cm-wide pots containing 18:9:1 play sand-gravel-Lansing soil (Hong et al. 2012) double autoclaved with a play sand layer in the middle of the pot containing either $500 \mathrm{G}$. versiforme spores, or a mock inoculum (Liu et al. 2004). After 2 weeks, two seedlings were culled from the pot, leaving only two plants of equal size. Plants were grown for 10 weeks and were fertilized with $50 \mathrm{ml}$ of $1 / 2 \times$ Hoagland's, $1 / 2 \times \mathrm{N}$, and $200 \mu \mathrm{M}$ Pi once a week for 9 weeks, with the exception of week 8 , to encourage a growth response. In addition to the Hoagland's fertilizer, plants were treated with $1 \mathrm{ml}$ of $10 \mathrm{mM} \mathrm{Ca}_{3}\left(\mathrm{PO}_{4}\right)_{2}$ insoluble Pi solution once a week, because this form of $\mathrm{Pi}$ is inaccessible to plants lacking AM fungal colonization. After 10 weeks, plants were harvested and fresh shoot weight, dry shoot weight, and fecundity were measured per pot.

\section{M. truncatula Pi fertilizer treatment.}

The M. truncatula Pi fertilizer treatment experiment was performed as described above, except plants were treated with $20 \mathrm{ml}$ of $1 / 2 \times$ Hoagland's and $1 / 2 \times \mathrm{N}$ containing either $20 \mu \mathrm{M}, 200 \mu \mathrm{M}$, or $2 \mathrm{mM}$ potassium phosphate twice a week 1 week after planting. Plants were harvested 3 weeks postplanting.

\section{$\mathrm{GA}_{3}$ treatment.}

$\mathrm{GA}_{3}$ treatment was performed as described by Floss et al. (2013a) but with the following modifications. Plants were grown in cones as described in the plant growth methods, and treated with $20 \mathrm{ml}$ of $\mathrm{GA}_{3}$ at $10^{-8}, 10^{-7}$, and $10^{-6} \mathrm{M}$ daily beginning at 6 days after planting.

\section{L. japonicus plant growth and Pi fertilizer treatment.}

For the L. japonicus Pi fertilizer treatment, L. japonicus seed were surface sterilized and planted as described for M. truncatula. Plants were treated with $20 \mathrm{ml}$ of $1 / 2 \times$ Hoagland's and $1 / 2 \times \mathrm{N}$ containing either 0,20 , or $200 \mu \mathrm{M}$ Pi twice a week beginning 1 week after planting. Plants were harvested 5 weeks postplanting.

\section{Tnt1 insertion lines and generation} of ipd3-2 ipd3l-2 double mutant.

We obtained Tnt1 insertion lines NF5939 (Mtipd3) and NF13859 (Mtipd3l) containing a Tnt1 insertion in either MtIPD3 (Medtr5g026850) or MtIPD3L (Medtr8g071320), respectively. The ipd3-2 Tnt1 mutant was verified by amplifying the Tntl insertion border using the Tntl border primer B1384 and IPD3-specific primer B5907, followed by PCR purification and sequencing. For ipd3l-2, the Tnt 1 border primer B2501 and IPD3L-specific primer B5368 were used to amplify the insertion border, and the PCR product was purified and sequenced.

After confirming the presence of the Tnt1 insertion, ipd3-2 and ipd $3 l-2$ were crossed. The $\mathrm{F}_{1}$ generation, consisting of eight individuals, was allowed to self pollinate, and $\mathrm{F}_{2}$ seed were collected. In all, $90 \mathrm{~F}_{2}$ plants were screened to identify homozygous WT, single, and double mutants via PCR-based screening of the Tnt 1 insertions. For screening for the presence of the IPD3 WT sequence, the primers B2110 and B5907 were used (492-bp product). For the ipd3 mutant screen, B1384 and B5907 were used (960-bp product). To screen IPD3L, B5367 and B5909 primers were used to screen the WT sequence $(2.2-\mathrm{kb}$ product). B2501 and B5909 were used to screen for the ipd3l
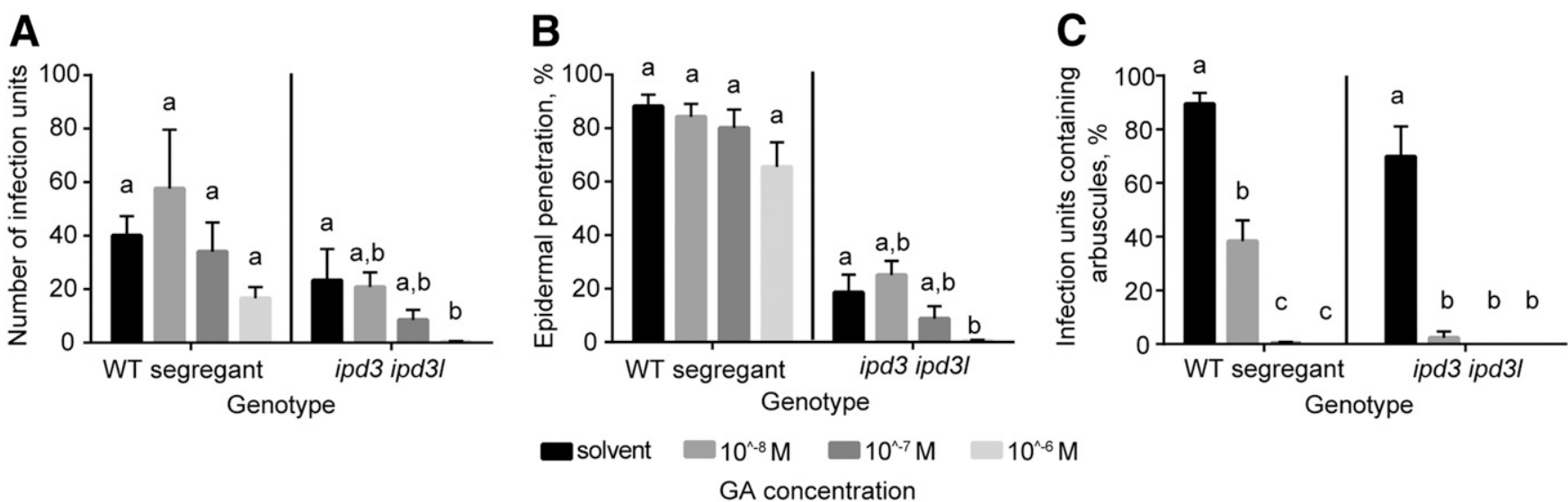

Fig. 7. Colonization of ipd3-2 ipd3l-2 shows increased sensitivity to GA 3 . Wild-type (WT) segregant and ipd3-2 ipd3l-2 plants were colonized with Glomus versiforme and treated with $\mathrm{GA}_{3}$. $\mathbf{A}$, Number of infection units; $\mathbf{B}$, percentage of hyphopodia that penetrate the epidermis; $\mathbf{C}$, percentage of infection units containing arbuscules. Error bars indicate standard error of the mean, $n=5$. Different letters signify statistically significant differences within genotypes from one-way analysis of variance, $P<0.05$, Tukey's honestly significant difference. 
sequence (620-bp product). The IPD3LWT gene was amplified and cloned into pGEM T-EASY using the primers B5367, located at the 5' ATG start site, and q2131, adjacent to the 3' TAG stop codon, then sequenced using the M13 primers B874 and B875. The IPD3L CDS was amplified and cloned into pDONR1-2 using primers B6199 and B6200 and then sequenced using B874 and B875.

Four independent homozygous double mutant lines, one homozygote for each single mutant, and three homozygous WT segregants were obtained and used in subsequent analyses.

\section{Plasmid construction.}

$I P D 3$ and IPD3L CDS were amplified from cDNA generated from A17 roots colonized by $G$. versiforme and cloned into pDONR1-2 plasmids. The primers used for amplification were as follows: B3759 and B3760 for IPD3, and B6199 and B6200 for IPD3L.

To clone autoactive versions of IPD3 and IPD3L, overlapping PCR was performed using the pDONR1-2 IPD3 and IPD $3 L$ CDS plasmids as a template. To generate amino acid substitutions, we used the following primers: for IPD3, B6236 and B6237 (S50D) and B6232 and B6233 (S154D); for IPD3L, B6234 and B6235 (S50D) and B6230 and B6231 (S154D). For the overlapping PCR, two sequential rounds of PCR were performed. For the first round, PCR was performed using the attB1F primer of either IPD3 or IPD3L and the reverse primer containing the amino acid substitution (e.g., B3759 and B6237 for IPD3 S50D), as well as the forward primer containing the amino acid substitution (B6236 and B3760 for IPD3 S50D) plus the attB2R reverse primer. After these two PCR products were generated, they were gel purified and simultaneously used as a template for the second PCR using the attB1F and attBR2 primers to generate a single PCR product. This overlapping PCR product was then gel extracted, cloned into pDONR1-2, and sequenced to ensure that the point mutation was introduced into the CDS.

The promoter region from a constitutively expressed ubiquitin, Medtr8g018230, was selected to overexpress IPD3 and IPD3L using expression profile information from the M. truncatula gene atlas. A 2-kb region upstream of the CDS was selected for the MtUBQpr. The promoter region was amplified from A17 genomic DNA with primers B6281 and B6282 containing attB4F and attB1R sites, respectively, and cloned into pDONR 4-1 using Gateway BP Clonase (Invitrogen).

To generate the GOF-DMI3 construct, DMI3 T265D plasmids were used as a template (Gleason et al. 2006). The primers B6360 and B6361 were used to amplify the truncated version of the sequence, and B6361 also contained an NLS (ATCCTC AACCTTTCTCTTCTTCTTAGG). Then, this PCR product was amplified using the primers B6360 and B6363 to incorporate a stop codon and attB2R site to the $3^{\prime}$ end of the CDS sequence.

To generate expression vectors for this study, pDONR plasmids were recombined into pKM43GW using a multisite reaction with Gateway LR Clonase (Invitrogen) (Supplementary Table S1).

For the $\mathrm{Y} 2 \mathrm{H}$ screen, IPD $3 L$ was cloned into the activation domain-containing plasmid pJG4-5 GW using a single-site LR reaction. The other $\mathrm{Y} 2 \mathrm{H}$ plasmids were previously cloned as described by Park et al. (2015).

\section{Agrobacterium rhizogenes-mediated transformation of $M$. truncatula roots.}

Root transformations were conducted as described by Boisson-Dernier et al. (2001), with the following modifications. Plants were grown on modified $\mathrm{F}$ plates in the absence of antibiotic, and transgenic roots were identified by the presence of DsRED or GFP, depending on the construct transformed (Floss et al. 2013b). Plants were grown in cones as described above and harvested 3 weeks postplanting, unless otherwise described. The presence of DsRED or GFP transgenic roots was again assessed immediately before harvesting to ensure that transgenic material was obtained for gene expression.

\section{Assessment of colonization via microscopy.}

Immediately after harvesting, roots were placed in $50 \%$ ethanol for $2 \mathrm{~h}$, after which they were transferred to $20 \%$ (wt/vol) $\mathrm{KOH}$, incubated for 3 days at $65^{\circ} \mathrm{C}$, then rinsed five times with double-distilled $\mathrm{H}_{2} \mathrm{O}$ with gentle agitation. After the final water rinse, roots were rinsed in phosphate-buffered saline (PBS) for a minimum of $30 \mathrm{~min}$, then stained with a 1: 10,000 WGA-PBS solution overnight at $4^{\circ} \mathrm{C}$. WGA-stained roots were examined using an Olympus SZX stereomicroscope, where the number and presence of fungal structures was analyzed, including the number of infection units, infection units containing arbuscules, and aborted hyphopodia on the surface of the root. Root length colonization was assayed using a modified grid-line intersect method (McGonigle et al. 1990). For confocal microscopy, roots were counterstained with propidium iodide at $20 \mu \mathrm{g} \mathrm{ml} \mathrm{m}^{-1}$ to observe plant cell wall material. Roots were imaged using an SP5 Leica confocal microscope as described previously (Ivanov and Harrison 2014).

\section{RNA isolation and gene expression analysis.}

RNA was isolated using the TRIzol protocol according to the manufacturer's procedure (Invitrogen). RNA (250 ng) was used to synthesize cDNA using Super Script Transcriptase III (Invitrogen). For qPCR analyses, $10 \mathrm{ng}$ of cDNA template was used. qPCR analyses were performed using an Applied Biosystems QuantFlex Studio 6.0 machine.

\section{Y2H assay.}

The $\mathrm{Y} 2 \mathrm{H}$ assay was conducted as described by Park et al. (2015).

\section{Statistical analysis.}

Statistical analyses were performed in R Studio using the -stats and -lsmeans statistical packages. A Student's $t$ test was performed for the experiments indicated. For one-way analysis of variance, Tukey's honestly significant difference analysis was performed and pairwise comparisons were determined. For gene expression analysis, nonparametric pairwise comparisons were obtained using a Kruskal-Wallis test.

\section{Phylogenetic analysis.}

Phylogenetic analyses were performed as previously described by Bravo et al. (2016).

\section{Synteny analysis.}

To perform the synteny analysis, we used the CoGe website platform. To compare LjCYCLOPS and MtIPD3, we entered their corresponding gene IDs to be able to map the surrounding chromosomal regions. At the time of the synteny analysis, IPD3L had not been annotated as a gene; therefore, we used the neighboring gene ID Medtr8g071330 to map the IPD3 and IPD3L chromosomal regions.

\section{Nodulation experiments.}

To assess the nodulation phenotype of ipd3-2 ipd3l-2, seed were sterilized as described above and planted into cones with 
two plants per cone containing the $2: 2: 1$ play sand-black sandgravel mixture previously described. Nodulation assays were performed with $S$. meliloti strain 1021 or nifHpr:GFP as previously described by Zhang et al. (2015b), with the following modifications. Plants were fertilized with $1 / 2 \times$ Hoagland's and $200 \mu \mathrm{M}$ Pi lacking N twice a week. Plants were harvested either 23 days (plants inoculated with $S$. meliloti 1021) or 28 days (S. meliloti nifHpr:GFP) postplanting.

Nodules and infection threads were counted and imaged using a Leica M205 stereoscope, and nodules were longitudinally sectioned and imaged using a Leica SP5 confocal microscope to observe symbiosomes.

\section{Primers.}

Primers for sequencing, cloning, and qPCR are listed in Supplementary Tables 2, 3, and 4.

\section{ACKNOWLEDGMENTS}

We thank G. Oldroyd the ipd3l-2 seed, L. Mueller for identifying the M. truncatula ubiquitin promoter used for this study, and L. Mueller and A. Bravo for their thoughtful review of the manuscript.

\section{AUTHOR-RECOMMENDED INTERNET RESOURCE}

CoGe website platform: https://genomevolution.org/coge/

\section{LITERATURE CITED}

Akiyama, K., Matsuzaki, K., and Hayashi, H. 2005. Plant sesquiterpenes induce hyphal branching in arbuscular mycorrhizal fungi. Nature 435 : 824-827.

Amijee, F., Tinker, P., and Stribley, D. 1989. The development of endomycorrhizal root systems VII. A detailed study of effects of soil phosphorus on colonization. New Phytol. 111:435-446.

Balzergue, C., Chabaud, M., Barker, D. G., Bécard, G., and Rochange, S. F. 2013. High phosphate reduces host ability to develop arbuscular mycorrhizal symbiosis without affecting root calcium spiking responses to the fungus. Front. Plant Sci. 4:426.

Balzergue, C., Puech-Pagès, V., Bécard, G., and Rochange, S. F. 2011. The regulation of arbuscular mycorrhizal symbiosis by phosphate in pea involves early and systemic signalling events. J. Exp. Bot. 62: 1049-1060.

Boisson-Dernier, A., Chabaud, M., Garcia, F., Bécard, G., Rosenberg, C., and Barker, D. G. 2001. Agrobacterium rhizogenes-transformed roots of Medicago truncatula for the study of nitrogen-fixing and endomycorrhizal symbiotic associations. Mol. Plant-Microbe Interact. 14:695-700.

Bradbury, S., Peterson, R., and Bowley, S. 1991. Interactions between three alfalfa nodulation genotypes and two Glomus species. New Phytol. 119: 115-120.

Branscheid, A., Sieh, D., Pant, B. D., May, P., Devers, E. A., Elkrog, A., Schauser, L., Scheible, W.-R., and Krajinski, F. 2010. Expression pattern suggests a role of MiR399 in the regulation of the cellular response to local Pi increase during arbuscular mycorrhizal symbiosis. Mol. PlantMicrobe Interact. 23:915-926.

Bravo, A., York, T., Pumplin, N., Mueller, L. A., and Harrison, M. J. 2016. Genes conserved for arbuscular mycorrhizal symbiosis identified through phylogenomics. Nat. Plants 2: Article 15208.

Breuillin, F., Schramm, J., Hajirezaei, M., Ahkami, A., Favre, P., Druege, U., Hause, B., Bucher, M., Kretzschmar, T., Bossolini, E., Kuhlemeier, C., Martinoia, E., Franken, P., Scholz, U., and Reinhardt, D. 2010. Phosphate systemically inhibits development of arbuscular mycorrhiza in Petunia hybrida and represses genes involved in mycorrhizal functioning. Plant J. 64:1002-1017.

Buee, M., Rossignol, M., Jauneau, A., Ranjeva, R., and Bécard, G. 2000. The pre-symbiotic growth of arbuscular mycorrhizal fungi is induced by a branching factor partially purified from plant root exudates. Mol. Plant-Microbe Interact. 13:693-698.

Burleigh, S. H., and Harrison, M. J. 1999. The down-regulation of Mt4-like genes by phosphate fertilization occurs systemically and involves phosphate translocation to the shoots. Plant Physiol. 119:241-248.

Catoira, R., Galera, C., de Billy, F., Penmetsa, R. V., Journet, E.-P., Maillet, F., Rosenberg, C., Cook, D., Gough, C., and Dénarié, J. 2000. Four genes of Medicago truncatula controlling components of a nod factor transduction pathway. Plant Cell 12:1647-1666.

Cerri, M. R., Wang, Q., Stolz, P., Folgmann, J., Frances, L., Katzer, K., Li, X., Heckmann, A. B., Wang, T. L., Downie, J. A., Klingl, A., de Carvalho-Niebel, F., Xie, F., and Parniske, M. 2017. The ERN1 transcription factor gene is a target of the CCaMK/CYCLOPS complex and controls rhizobial infection in Lotus japonicus. New Phytol. 215: 323-337.

Charpentier, M., Bredemeier, R., Wanner, G., Takeda, N., Schleiff, E., and Parniske, M. 2008. Lotus japonicus CASTOR and POLLUX are ion channels essential for perinuclear calcium spiking in legume root endosymbiosis. Plant Cell 20:3467-3479.

Charpentier, M., Sun, J., Vaz Martins, T., Radhakrishnan, G. V., Findlay, K., Soumpourou, E., Thouin, J., Véry, A.-A., Sanders, D., Morris, R. J., and Oldroyd, G. E. 2016. Nuclear-localized cyclic nucleotide-gated channels mediate symbiotic calcium oscillations. Science 352:1102-1105.

Chen, C., Ané, J. M., and Zhu, H. 2008. OsIPD3, an ortholog of the Medicago truncatula DMI3 interacting protein IPD3, is required for mycorrhizal symbiosis in rice. New Phytol. 180:311-315.

Davière, J. M., and Achard, P. 2013. Gibberellin signaling in plants. Development 140:1147-1151.

Delaux, P. M., Varala, K., Edger, P. P., Coruzzi, G. M., Pires, J. C., and Ané, J. M. 2014. Comparative phylogenomics uncovers the impact of symbiotic associations on host genome evolution. PLoS Genet. 10:e1004487.

Endre, G., Kereszt, A., Kevei, Z., Mihacea, S., Kaló, P., and Kiss, G. B 2002. A receptor kinase gene regulating symbiotic nodule development. Nature 417:962-966.

Floss, D. S., Levy, J. G., Lévesque-Tremblay, V., Pumplin, N., and Harrison, M. J. 2013a. DELLA proteins regulate arbuscule formation in arbuscular mycorrhizal symbiosis. Proc. Natl. Acad. Sci. U.S.A. 110:E5025-E5034.

Floss, D. S., Schmitz, A. M., Starker, C. G., Gantt, J. S., and Harrison, M. J. 2013b. Gene silencing in Medicago truncatula roots using RNAi. Methods Mol. Biol. 1069:163-177.

Genre, A., Chabaud, M., Balzergue, C., Puech-Pagès, V., Novero, M., Rey, T., Fournier, J., Rochange, S., Bécard, G., Bonfante, P., and Barker, D. G. 2013. Short-chain chitin oligomers from arbuscular mycorrhizal fungi trigger nuclear $\mathrm{Ca}^{2+}$ spiking in Medicago truncatula roots and their production is enhanced by strigolactone. New Phytol. 198:190-202.

Gleason, C., Chaudhuri, S., Yang, T., Muñoz, A., Poovaiah, B. W., and Oldroyd, G. E. 2006. Nodulation independent of rhizobia induced by a calcium-activated kinase lacking autoinhibition. Nature 441:1149-1152.

Gutjahr, C., Banba, M., Croset, V., An, K., Miyao, A., An, G., Hirochika H., Imaizumi-Anraku, H., and Paszkowski, U. 2008. Arbuscular mycorrhiza-specific signaling in rice transcends the common symbiosis signaling pathway. Plant Cell 20:2989-3005.

Gutjahr, C., and Parniske, M. 2013. Cell and developmental biology of arbuscular mycorrhiza symbiosis. Annu. Rev. Cell Dev. Biol. 29: 593-617.

Hayashi, T., Shimoda, Y., Sato, S., Tabata, S., Imaizumi-Anraku, H., and Hayashi, M. 2014. Rhizobial infection does not require cortical expression of upstream common symbiosis genes responsible for the induction of $\mathrm{Ca}^{2+}$ spiking. Plant J. 77:146-159.

Hong, J. J., Park, Y.-S., Bravo, A., Bhattarai, K. K., Daniels, D. A., and Harrison, M. J. 2012. Diversity of morphology and function in arbuscular mycorrhizal symbioses in Brachypodium distachyon. Planta 236: 851-865.

Horváth, B., Yeun, L. H., Domonkos, A., Halász, G., Gobbato, E., Ayaydin, F., Miró, K., Hirsch, S., Sun, J., Tadege, M., Ratet, P., Mysore, K. S., Ané, J. M., Oldroyd, G. E., and Kaló, P. 2011. Medicago truncatula IPD3 is a member of the common symbiotic signaling pathway required for rhizobial and mycorrhizal symbioses. Mol. Plant-Microbe Interact. 24: 1345-1358.

Ivanov, S., and Harrison, M. J. 2014. A set of fluorescent protein-based markers expressed from constitutive and arbuscular mycorrhiza-inducible promoters to label organelles, membranes and cytoskeletal elements in Medicago truncatula. Plant J. 80:1151-1163.

Jacobi, L. M., Zubkova, L. A., Barmicheva, E. M., Tsyganov, V. E., Borisov, A. Y., and Tikhonovich, I. A. 2003. Effect of mutations in the pea genes Sym33 and Sym40. II. Dynamics of arbuscule development and turnover. Mycorrhiza 13:9-16.

Jiang, C., Gao, X., Liao, L., Harberd, N. P., and Fu, X. 2007. Phosphate starvation root architecture and anthocyanin accumulation responses are modulated by the gibberellin-DELLA signaling pathway in Arabidopsis. Plant Physiol. 145:1460-1470.

Jin, Y., Chen, Z., Yang, J., Mysore, K. S., Wen, J., Huang, J., Yu, N., and Wang, E. 2018. IPD3 and IPD3L function redundantly in rhizobial and mycorrhizal symbioses. Front. Plant Sci. 9:267. 
Jin, Y., Liu, H., Luo, D., Yu, N., Dong, W., Wang, C., Zhang, X., Dai, H., Yang, J., and Wang, E. 2016. DELLA proteins are common components of symbiotic rhizobial and mycorrhizal signalling pathways. Nat. Commun. 7: Article 12433.

Kistner, C., Winzer, T., Pitzschke, A., Mulder, L., Sato, S., Kaneko, T., Tabata, S., Sandal, N., Stougaard, J., Webb, K. J., Szczyglowski, K., and Parniske, M. 2005. Seven Lotus japonicus genes required for transcriptional reprogramming of the root during fungal and bacterial symbiosis. Plant Cell 17:2217-2229.

Kobae, Y., Kameoka, H., Sugimura, Y., Saito, K., Ohtomo, R., Fujiwara, T., and Kyozuka, J. 2018. Strigolactone biosynthesis genes of rice are required for the punctual entry of arbuscular mycorrhizal fungi into the roots. Plant Cell Physiol. 59:544-553.

Lanfranco, L., Fiorilli, V., and Gutjahr, C. 2018. Partner communication and role of nutrients in the arbuscular mycorrhizal symbiosis. New Phytol. 220:1031-1046.

Larkan, N. J., Ruzicka, D. R., Edmonds-Tibbett, T., Durkin, J. M., Jackson, L. E., Smith, F. A., Schachtman, D. P., Smith, S. E., and Barker, S. J. 2013. The reduced mycorrhizal colonisation ( $\mathrm{rmc}$ ) mutation of tomato disrupts five gene sequences including the CYCLOPS/IPD3 homologue. Mycorrhiza 23:573-584.

Lévy, J., Bres, C., Geurts, R., Chalhoub, B., Kulikova, O., Duc, G., Journet, E.-P., Ané, J.-M., Lauber, E., Bisseling, T., Dénarié, J., Rosenberg, C., and Debellé, F. 2004. A putative $\mathrm{Ca}^{2+}$ and calmodulin-dependent protein kinase required for bacterial and fungal symbioses. Science 303: 1361-1364.

Liu, J., Blaylock, L. A., and Harrison, M. J. 2004. cDNA arrays as a tool to identify mycorrhiza-regulated genes: Identification of mycorrhizainduced genes that encode or generate signaling molecules implicated in the control of root growth. Can. J. Bot. 82:1177-1185.

MacLean, A. M., Bravo, A., and Harrison, M. J. 2017. Plant signaling and metabolic pathways enabling arbuscular mycorrhizal symbiosis. Plant Cell 29:2319-2335.

Maillet, F., Poinsot, V., André, O., Puech-Pagès, V., Haouy, A., Gueunier, M., Cromer, L., Giraudet, D., Formey, D., Niebel, A., Martinez, E. A., Driguez, H., Bécard, G., and Dénarié, J. 2011. Fungal lipochitooligosaccharide symbiotic signals in arbuscular mycorrhiza. Nature 469: 58-63.

McGonigle, T., Miller, M., Evans, D., Fairchild, G., and Swan, J. 1990. A new method which gives an objective measure of colonization of roots by vesicular-arbuscular mycorrhizal fungi. New Phytol. 115: 495-501.

Messinese, E., Mun, J.-H., Yeun, L. H., Jayaraman, D., Rougé, P., Barre, A., Lougnon, G., Schornack, S., Bono, J.-J., Cook, D. R., and Ané, J. M. 2007. A novel nuclear protein interacts with the symbiotic DMI3 calcium- and calmodulin-dependent protein kinase of Medicago truncatula. Mol. Plant-Microbe Interact. 20:912-921.

Mitra, R. M., Gleason, C. A., Edwards, A., Hadfield, J., Downie, J. A., Oldroyd, G. E., and Long, S. R. 2004. A Ca ${ }^{2+} /$ calmodulin-dependent protein kinase required for symbiotic nodule development: Gene identification by transcript-based cloning. Proc. Natl. Acad. Sci. U.S.A. 101:4701-4705.

Miyata, K., Kozaki, T., Kouzai, Y., Ozawa, K., Ishii, K., Asamizu, E., Okabe, Y., Umehara, Y., Miyamoto, A., Kobae, Y., Akiyama, K., Kaku, H., Nishizawa, Y., Shibuya, N., and Nakagawa, T. 2014. The bifunctional plant receptor, OsCERK1, regulates both chitin-triggered immunity and arbuscular mycorrhizal symbiosis in rice. Plant Cell Physiol. 55: 1864-1872.

Mosse, B. 1973. Plant growth responses to vesicular-arbuscular mycorrhiza. New Phytol. 72:127-136.

Oldroyd, G. E. 2013. Speak, friend, and enter: Signalling systems that promote beneficial symbiotic associations in plants. Nat. Rev. Microbiol. 11:252-263.

Ovchinnikova, E., Journet, E.-P., Chabaud, M., Cosson, V., Ratet, P., Duc, G., Fedorova, E., Liu, W., den Camp, R. O., Zhukov, V., Tikhonovich, I., Borisov, A., Bisseling, T., and Limpens, E. 2011. IPD3 controls the formation of nitrogen-fixing symbiosomes in pea and Medicago spp. Mol. Plant-Microbe Interact. 24:1333-1344.

Park, H. J., Floss, D. S., Levesque-Tremblay, V., Bravo, A., and Harrison, M. J. 2015. Hyphal branching during arbuscule development requires reduced arbuscular mycorrhiza1. Plant Physiol. 169:2774-2788.

Pimprikar, P., Carbonnel, S., Paries, M., Katzer, K., Klingl, V., Bohmer, M. J., Karl, L., Floss, D. S., Harrison, M. J., Parniske, M., and Gutjahr, C. 2016. A CCaMK-CYCLOPS-DELLA complex activates transcription of RAM1 to regulate arbuscule branching. Curr. Biol. 26:987-998.

Pumplin, N., Mondo, S. J., Topp, S., Starker, C. G., Gantt, J. S., and Harrison, M. J. 2010. Medicago truncatula Vapyrin is a novel protein required for arbuscular mycorrhizal symbiosis. Plant J. 61:482-494.
Riely, B. K., Lougnon, G., Ané, J. M., and Cook, D. R. 2007. The symbiotic ion channel homolog DMI1 is localized in the nuclear membrane of Medicago truncatula roots. Plant J. 49:208-216.

Saito, K., Yoshikawa, M., Yano, K., Miwa, H., Uchida, H., Asamizu, E., Sato, S., Tabata, S., Imaizumi-Anraku, H., Umehara, Y., Kouchi, H., Murooka, Y., Szczyglowski, K., Downie, J. A., Parniske, M., Hayashi, M., and Kawaguchi, M. 2007. NUCLEOPORIN85 is required for calcium spiking, fungal and bacterial symbioses, and seed production in Lotus japonicus. Plant Cell 19:610-624.

Silverstone, A. L., Jung, H.-S., Dill, A., Kawaide, H., Kamiya, Y., and Sun, T. P. 2001. Repressing a repressor: Gibberellin-induced rapid reduction of the RGA protein in Arabidopsis. Plant Cell 13:1555-1566.

Singh, S., Katzer, K., Lambert, J., Cerri, M., and Parniske, M. 2014 CYCLOPS, a DNA-binding transcriptional activator, orchestrates symbiotic root nodule development. Cell Host Microbe 15:139-152.

Singh, S., and Parniske, M. 2012. Activation of calcium- and calmodulindependent protein kinase (CCaMK), the central regulator of plant root endosymbiosis. Curr. Opin. Plant Biol. 15:444-453.

Smith, S. E., and Read, D. J. 2010. Mycorrhizal Symbiosis. Academic Press Elsevier Press. London.

Tadege, M., Wen, J., He, J., Tu, H., Kwak, Y., Eschstruth, A., Cayrel, A., Endre, G., Zhao, P. X., Chabaud, M., Ratet, P., and Mysore, K. S. 2008. Large-scale insertional mutagenesis using the Tnt1 retrotransposon in the model legume Medicago truncatula. Plant J. 54:335-347.

Takeda, N., Handa, Y., Tsuzuki, S., Kojima, M., Sakakibara, H., and Kawaguchi, M. 2015. Gibberellins interfere with symbiosis signaling and gene expression and alter colonization by arbuscular mycorrhizal fungi in Lotus japonicus. Plant Physiol. 167:545-557.

Takeda, N., Maekawa, T., and Hayashi, M. 2012. Nuclear-localized and deregulated calcium- and calmodulin-dependent protein kinase activates rhizobial and mycorrhizal responses in Lotus japonicus. Plant Cell 24: 810-822.

Tawaraya, K., Hashimoto, K., and Wagatsuma, T. 1998. Effect of root exudate fractions from P-deficient and P-sufficient onion plants on roo colonisation by the arbuscular mycorrhizal fungus Gigaspora margarita. Mycorrhiza 8:67-70.

Thomson, B., Robson, A., and Abbott, L. 1986. Effects of phosphorus on the formation of mycorrhizas by Gigaspora calospora and Glomus fasciculatum in relation to root carbohydrates. New Phytol. 103:751-765.

Venkateshwaran, M., Jayaraman, D., Chabaud, M., Genre, A., Balloon, A. J., Maeda, J., Forshey, K., den Os, D., Kwiecien, N. W., Coon, J. J., Barker, D. G., and Ané, J. M. 2015. A role for the mevalonate pathway in early plant symbiotic signaling. Proc. Natl. Acad. Sci. U.S.A. 112:97819786.

Xiao, T. T., Schilderink, S., Moling, S., Deinum, E. E., Kondorosi, E., Franssen, H., Kulikova, O., Niebel, A., and Bisseling, T. 2014. Fate map of Medicago truncatula root nodules. Development 141:3517-3528.

Yano, K., Yoshida, S., Müller, J., Singh, S., Banba, M., Vickers, K., Markmann, K., White, C., Schuller, B., Sato, S., Asamizu, E., Tabata, S., Murooka, Y., Perry, J., Wang, T. L., Kawaguchi, M., Imaizumi-Anraku, H., Hayashi, M., and Parniske, M. 2008. CYCLOPS, a mediator of symbiotic intracellular accommodation. Proc. Natl. Acad. Sci. U.S.A. 105:20540-20545.

Young, N. D., Debellé, F., Oldroyd, G. E., Geurts, R., Cannon, S. B., Udvardi, M. K., Benedito, V. A., Mayer, K. F., Gouzy, J., Schoof, H., Van de Peer, Y., Proost, S., Cook, D. R., Meyers, B. C., Spannagl, M., Cheung, F., De Mita, S., Krishnakumar, V., Gundlach, H., Zhou, S., Mudge, J., Bharti, A. K., Murray, J. D., Naoumkina, M. A., Rosen, B., Silverstein, K. A., Tang, H., Rombauts, S., Zhao, P. X., Zhou, P., Barbe, V., Bardou, P., Bechner, M., Bellec, A., Berger, A., Bergès, H., Bidwell, S., Bisseling, T., Choisne, N., Couloux, A., Denny, R., Deshpande, S. Dai, X., Doyle, J. J., Dudez, A. M., Farmer, A. D., Fouteau, S., Franken, C., Gibelin, C., Gish, J., Goldstein, S., González, A. J., Green, P. J., Hallab, A., Hartog, M., Hua, A., Humphray, S. J., Jeong, D. H., Jing, Y., Jöcker, A., Kenton, S. M., Kim, D. J., Klee, K., Lai, H., Lang, C., Lin, S., Macmil, S. L., Magdelenat, G., Matthews, L., McCorrison, J., Monaghan, E. L., Mun, J. H., Najar, F. Z., Nicholson, C., Noirot, C., O’Bleness, M., Paule, C. R., Poulain, J., Prion, F., Qin, B., Qu, C., Retzel, E. F., Riddle, C., Sallet, E., Samain, S., Samson, N., Sanders, I., Saurat, O., Scarpelli, C., Schiex, T., Segurens, B., Severin, A. J., Sherrier, D. J., Shi, R., Sims, S., Singer, S. R., Sinharoy, S., Sterck, L., Viollet, A., Wang, B. B., Wang, K., Wang, M., Wang, X., Warfsmann, J., Weissenbach, J., White, D. D., White, J. D., Wiley, G. B., Wincker, P., Xing, Y., Yang, L., Yao, Z., Ying, F., Zhai, J., Zhou, L., Zuber, A., Dénarié, J., Dixon, R. A., May, G. D., Schwartz, D. C., Rogers, J., Quétier, F., Town, C. D., and Roe, B. A. 2011. The Medicago genome provides insight into the evolution of rhizobial symbioses. Nature 480: 520-524. 
Zentella, R., Hu, J., Hsieh, W.-P., Matsumoto, P. A., Dawdy, A., Barnhill, B., Oldenhof, H., Hartweck, L. M., Maitra, S., Thomas, S. G., Cockrell, S., Boyce, M., Shabanowitz, J., Hunt, D. F., Olszewski, N. E., and Sun, T. P. 2016. O-GlcNAcylation of master growth repressor DELLA by SECRET AGENT modulates multiple signaling pathways in Arabidopsis. Genes Dev. 30:164-176.
Zhang, X., Dong, W., Sun, J., Feng, F., Deng, Y., He, Z., Oldroyd, G. E., and Wang, E. 2015a. The receptor kinase CERK1 has dual functions in symbiosis and immunity signalling. Plant J. 81:258-267.

Zhang, X., Pumplin, N., Ivanov, S., and Harrison, M. J. 2015b. EXO70I is required for development of a sub-domain of the periarbuscular membrane during arbuscular mycorrhizal Symbiosis. Curr. Biol. 25:2189-2195. 